\title{
ERGODIC FREQUENCY MEASURES FOR RANDOM SUBSTITUTIONS
}

\author{
PHILIPP GOHLKE AND TIMO SPINDELER
}

\begin{abstract}
We construct a family of ergodic measures on random substitution subshifts (RSsubshifts) associated to a primitive random substitution. In particular, the word frequencies of every finite legal word exist for almost every element of the random substitution subshift with respect to these measures. As an application, we show that for a certain class of random substitutions the measures of maximal entropy are frequency measures.
\end{abstract}

\section{INTRODUCTION}

Deterministic substitutions and the corresponding dynamical systems are well-studied objects in symbolic dynamics, which give rise to applications in other branches of mathematics and science $[2,24,25]$. Early efforts to understand the properties of randomised versions of these substitutions were made in a pioneering work by Peyrière [23]. Later, the concept of random substitutions also evoked interest in the physics community [7, 12]. Over the decades, random substitutions have appeared in various disguises and contexts, including $0 L$-systems in formal language theory [26] and expansion-modification systems [15] that where used to model long-term correlation decay in DNA sequences [29, 17]. However, a systematic study of random substitutions is still in its infancy. Some basic topological properties were studied in [28], a study of periodic points associated to random substitution subshifts was undertaken in [27]. When passing to the associated subshift, it is desirable to construct measures that keep the structure of the underlying stochastic process meaningful, stressing the point of view that it can be considered as some limiting object of the iterated random substitution. Recently, for a certain class of random substitutions, equilibrium measures have been constructed that are invariant under the substitution action [16]. In this work, we are concerned with measures which are shift-invariant, a property which is usually taken as a starting point for assigning objects like diffraction measures or the spectrum of a Schrödinger operator to the subshift. In diffraction theory, random substitutions are used to generalise results from the deterministic setting $[4,32]$. For instance, it is a well-known fact that a point set which comes from certain primitive substitutions gives rise to a pure point diffraction measure, and, consequently, to a pure point dynamical spectrum [3]. In contrast, in the case of randomised versions of these substitutions, one may obtain a mixed diffraction spectrum and a mixed dynamical spectrum, consisting of a pure point part and an absolutely continuous part [19,4]. The ergodicity of the associated measures plays a vital role to ensure that this diffraction spectrum is the same

2010 Mathematics Subject Classification. 37B10, 37A25, 37A50, 52C23.

Key words and phrases. Random substitutions, ergodic measures, measures of maximal entropy. 
for almost every realisation. Similarly, the generic choice of a Schrödinger spectrum as an almost-surely constant object, relies on the existence of an ergodic measure [5]. Therefore, ergodicity is a desirable property of measures that arise from random substitutions.

We are going to construct these measures in the following way. It is a well-known fact that, if $\rho$ is a primitive substitution, its hull $\mathbb{X}_{\rho}$ is strictly ergodic under the $\mathbb{Z}$-action of the shift [2, Thm. 4.3]. Moreover, if $x \in \mathbb{X}_{\rho}$ and $v$ is a subword of $x$, then $\mu([v])$ is given by the word frequency of $v$ in $x$ (which exists uniformly due to Oxtoby's theorem), where $\mu$ is the unique shift invariant and ergodic measure on $\mathbb{X}_{\rho}$ and $[v]$ is the cylinder set which corresponds to $v$. We would like to transfer this idea to random substitutions. Here, we are no longer in the strictly ergodic setting. Instead, we choose a measure $\mu$ that is compatible with the word frequencies that arise almost surely in the limit of large inflation words. We establish the ergodicity of $\mu$ by showing that the word frequencies of all legal words exist and coincide for $\mu$-almost all possible realisations. The ergodicity of the frequency measures arising from a certain class of random substitutions was previously claimed in [20, Thm. 4.22]. The proof, however, contains a small gap, since it relies on the independence of certain random words, which is only asymptotically true and thus requires some extra work.

The paper is organised as follows. In Section 2, we introduce random substitutions and describe them as Markov processes. Here, we follow the approach presented in [13], which was also implicitly used in [20]. The corresponding probability measure is used in Section 3 to establish well-defined word frequencies in expectation. In Section 4, we generalise this result to the almost-sure existence of word frequencies. From these, we construct a shift-invariant measure on the stochastic hull of the random substitution in Section 5, and show that it is ergodic, comprising the main result of this paper. In the last section, we compute the metric entropy of a class of frequency measures for an explicit example. Moreover, we show that for a certain class of random substitutions one of the frequency measures is indeed the measure of maximal entropy.

\section{Random Substitutions}

The definition of a random substitution, presented in this section, basically follows the notion of a substitution Markov chain, introduced under that name in [13] and going back to [23]. However, our notation is slightly different to parallel the one used for deterministic substitutions [2]. It is mainly in accordance with [20].

Given a finite alphabet $\mathcal{A}$, a word in $\mathcal{A}$ is any finite concatenation of symbols in $\mathcal{A}$. The length of a word $u$ is denoted by $|u|$. For a given word $u=u_{1} \cdots u_{n}$ and two positions $k, m \in \mathbb{N}$ with $1 \leqslant k \leqslant m \leqslant n$, we define $u_{[k, m]}=u_{k} \cdots u_{m}$. The number of occurrences of a word $v=v_{1} \cdots v_{k}$ within a word $u=u_{1} \cdots u_{n}$ is given by $|u|_{v}=\operatorname{card}\left\{0 \leqslant i \leqslant n-k: u_{[i+1, i+k]}=v\right\}$, which is 0 in case that $k>n$. If $|u|_{v} \geqslant 1$, we say that $v$ is a subword of $u$ and we write $v \triangleleft u$ in that situation. Let $\mathcal{A}^{+}=\cup_{n \geqslant 1} \mathcal{A}^{n}$ denote the set of finite words in $\mathcal{A}$. Assuming the alphabet is of size $m=\operatorname{card} \mathcal{A}$, the Abelianisation $\Phi$ is a map

$$
\Phi: \mathcal{A}^{+} \rightarrow \mathbb{R}^{m}, \quad \Phi(u)_{a}=|u|_{a}, \text { for all } a \in \mathcal{A} .
$$

Given $u, v \in \mathcal{A}^{+}$we write the concatenation of $u$ and $v$ as $u v$. To avoid confusion, we will index words by superscripts, that is, $u=u^{1} \cdots u^{m}$ denotes a concatenation of words 
$u^{1}, \ldots, u^{m} \in \mathcal{A}^{+}$, whereas $u=u_{1} \ldots u_{n}$ is the decomposition of $u$ into letters. We equip $\mathcal{A}^{+}$ with the discrete topology. Thus, $\mathcal{B}=\mathcal{P}\left(\mathcal{A}^{+}\right)$, with $\mathcal{P}\left(\mathcal{A}^{+}\right)$the power set of $\mathcal{A}^{+}$, gives the $\sigma$-algebra of Borel sets. We denote by $\mathcal{A}^{\mathbb{Z}}$ the set of all bi-infinite sequences in $\mathcal{A}$ and we set $x_{[k, m]}=x_{k} \cdots x_{m}$ for all $x \in \mathcal{A}^{\mathbb{Z}}$ and $k \leqslant m \in \mathbb{Z}$. The shift-action on $\mathcal{A}^{\mathbb{Z}}$ is defined via $(S x)_{i}=x_{i+1}$ for all $x \in \mathcal{A}^{\mathbb{Z}}$ and $i \in \mathbb{Z}$.

Definition 2.1. A random word on a probability space $(\Omega, \mathcal{F}, \mathbb{P})$ is a measurable function $\mathcal{U}:(\Omega, \mathcal{F}) \rightarrow\left(\mathcal{A}^{+}, \mathcal{P}\left(\mathcal{A}^{+}\right)\right)$. The distribution of $\mathcal{U}$ is the probability measure $\mathbb{P} \circ \mathcal{U}^{-1}$ on $\left(\mathcal{A}^{+}, \mathcal{P}\left(\mathcal{A}^{+}\right)\right)$. We call $u \in \mathcal{A}^{+}$a realisation of $\mathcal{U}$ if $\mathbb{P}[\mathcal{U}=u]:=\mathbb{P}\left(\mathcal{U}^{-1}(\{u\})\right)>0$.

Remark 2.2. Given a random word $\mathcal{U}$ on $(\Omega, \mathcal{F}, \mathbb{P})$, notions like length or Abelianisation that are defined for words naturally extend to actions on $\mathcal{U}$. More precisely, if $f: \mathcal{A}^{+} \rightarrow X$, for a measurable space $(X, \mathcal{G})$, we define

$$
f(\mathcal{U}):=f \circ \mathcal{U}: \Omega \rightarrow X .
$$

Note that $f(\mathcal{U})$ is measurable without further requirements because we have chosen $\mathcal{P}\left(\mathcal{A}^{+}\right)$ as the sigma-algebra of words. If $X=\mathbb{R}$, it thus defines a random variable on $(\Omega, \mathcal{F}, \mathbb{P})$. In particular, $|\mathcal{U}|$ is a well-defined random variable on $(\Omega, \mathcal{F}, \mathbb{P})$, and $\Phi(\mathcal{U})$ a random vector. Similarly, $\mathcal{U}_{1}$ is a random word (in fact a random letter) defined as $g \circ \mathcal{U}$, where $g: \mathcal{A}^{+} \rightarrow$ $\mathcal{A}, u_{1} \cdots u_{m} \mapsto u_{1}$.

Definition 2.3. If $\mathcal{U}$ and $\mathcal{V}$ are random words on the same probability space $(\Omega, \mathcal{F}, \mathbb{P})$, we define the concatenation of $\mathcal{U}$ and $\mathcal{V}$ as the random word

$$
\mathcal{U V}: \Omega \rightarrow \mathcal{A}^{+}, \quad(\mathcal{U V})(\omega)=\mathcal{U}(\omega) \mathcal{V}(\omega)
$$

We call $\mathcal{U V}$ an independent concatenation if $\mathcal{U}$ and $\mathcal{V}$ are independent random words, that is $\mathbb{P} \circ(\mathcal{U}, \mathcal{V})^{-1}=\mathbb{P} \circ \mathcal{U}^{-1} \otimes \mathbb{P} \circ \mathcal{V}^{-1}$ is the product measure on $\left(\mathcal{A}^{+}\right)^{2}$.

Remark 2.4. If $\mathcal{U}$ and $\mathcal{V}$ are defined on different probability spaces $\left(\Omega_{1}, \mathcal{F}_{1}, \mathbb{P}_{1}\right)$ and $\left(\Omega_{2}, \mathcal{F}_{2}, \mathbb{P}_{2}\right)$ respectively, we can still define an independent concatenation of $\mathcal{U}$ and $\mathcal{V}$ on the product space $\left(\Omega_{1} \times \Omega_{2}, \mathcal{F}_{1} \otimes \mathcal{F}_{2}, \mathbb{P}_{1} \otimes \mathbb{P}_{2}\right)$. This is obtained via $\mathcal{U} \mathcal{V}\left(\omega_{1}, \omega_{2}\right)=\mathcal{U}\left(\omega_{1}\right) \mathcal{V}\left(\omega_{2}\right)$.

The intuitive idea of a random substitution is the following. Given $u \in \mathcal{A}^{+}$, replace each letter in $u$ at random and independently by a word in $\mathcal{A}^{+}$. The distribution for such a replacement shall depend only on the type of the letter that is being replaced. We thus obtain a random word. If we condition on the event that this random word takes a specific value in $\mathcal{A}^{+}$, we can iterate the process. This leads to the structure of a stationary Markov chain with countable state space.

We proceed by introducing the data that is needed to define a random substitution. Let us denote by $\mathcal{M}_{f}\left(\mathcal{A}^{+}\right)$the space of probability measures on $\mathcal{A}^{+}$with finite support.

Definition 2.5. A random substitution rule on a finite alphabet $\mathcal{A}$ is a map

$$
P^{\vartheta}: \mathcal{A} \rightarrow \mathcal{M}_{f}\left(\mathcal{A}^{+}\right), \quad a \mapsto P_{a}^{\vartheta} .
$$

The corresponding transition kernel is a map $P: \mathcal{A}^{+} \times \mathcal{A}^{+} \rightarrow[0,1]$ with

$$
P\left(u_{1} \cdots u_{m}, v\right)=\sum_{\substack{v^{1}, \ldots, v^{m} \in \mathcal{A}^{+} \\ v^{1} \cdots v^{m}=v}} P_{u_{1}}^{\vartheta}\left(v^{1}\right) \cdots P_{u_{m}}^{\vartheta}\left(v^{m}\right) .
$$


In particular, $P(a, v)=P_{a}^{\vartheta}(v)$ for $a \in \mathcal{A}, v \in \mathcal{A}^{+}$and $P(u, \cdot)$ is a probability measure in $\mathcal{M}_{f}\left(\mathcal{A}^{+}\right)$for all $u \in \mathcal{A}^{+}$. In analogy to transition matrices, powers of the transition kernel $P$ are defined inductively via $P^{k+1}(u, v)=\sum_{w \in \mathcal{A}^{+}} P^{k}(u, w) P(w, v)$, for all $u, v \in \mathcal{A}^{+}$.

Remark 2.6. Let us expand a bit on the structure of the transition kernel. Suppose $\mathcal{U}_{1}, \ldots, \mathcal{U}_{m}$ are independent random words with distribution $P_{u_{1}}^{\vartheta}, \ldots, P_{u_{m}}^{\vartheta}$, respectively. Then, it is a straightforward calculation to verify that the distribution of $\mathcal{U}_{1} \cdots \mathcal{U}_{m}$ is given by $P\left(u_{1} \cdots u_{m}, \cdot\right)$. In other words, $P(u, \cdot)$ describes the distribution if each letter in $u$ is mapped independently to a random word, according to the random substitution rule and the resulting random words are concatenated in the same order as the original letters. It is worth mentioning that the independence assumption might be relaxed leading to a more general concept of a random substitution, coined $M$-system in [23]. This corresponds to a different choice of the transition kernel.

Given a random substitution rule $P^{\vartheta}$, we want to define a random substitution $\vartheta$ as a map between random words. There is a technical subtlety in that we need to enlarge the probability space of the original random word in order to make enough 'space' for the new one. Suppose $\mathcal{U}$ is a random word on $\left(\Omega_{1}, \mathcal{F}_{1}, \mathbb{P}_{1}\right)$ and let $\left(\Omega_{2}, \mathcal{F}_{2}, \mathbb{P}_{2}\right)$ be a second probability space. With slight abuse of notation we can extend $\mathcal{U}$ to a random variable on $\left(\Omega_{1} \times \Omega_{2}, \mathcal{F}_{1} \otimes \mathcal{F}_{2}, \mathbb{P}_{1} \otimes \mathbb{P}_{2}\right)$, via $\mathcal{U}\left(\left(\omega_{1}, \omega_{2}\right)\right):=\mathcal{U}\left(\omega_{1}\right)$ for all $\left(\omega_{1}, \omega_{2}\right) \in \Omega_{1} \times \Omega_{2}$. Note that $\left(\mathbb{P}_{1} \otimes \mathbb{P}_{2}\right) \circ \mathcal{U}^{-1}=\mathbb{P}_{1} \circ \mathcal{U}^{-1}$, so $\mathcal{U}$ induces the same distribution on $\mathcal{A}^{+}$in both cases.

Definition 2.7. Let $P^{\vartheta}$ be a random substitution rule with transition kernel $P$. A corresponding random substitution $\vartheta$ is a map between random words with the following properties. If $\mathcal{U}$ is a random word on $\left(\Omega_{1}, \mathcal{F}_{1}, \mathbb{P}_{1}\right)$ we define $\vartheta(\mathcal{U})$ to be a random word on $(\Omega, \mathcal{F}, \mathbb{P})=\left(\Omega_{1} \times \Omega_{2}, \mathcal{F}_{1} \otimes \mathcal{F}_{2}, \mathbb{P}_{1} \otimes \mathbb{P}_{2}\right)$ for some probability space $\left(\Omega_{2}, \mathcal{F}_{2}, \mathbb{P}_{2}\right)$, satisfying

$$
\mathbb{P}[\vartheta(\mathcal{U})=v \mid \mathcal{U}=u]=P(u, v),
$$

for all $u, v \in \mathcal{A}^{+}$.

Remark 2.8. For a fixed $P^{\vartheta}$, there is some freedom to choose the probability space $\left(\Omega_{2}, \mathcal{F}_{2}, \mathbb{P}_{2}\right)$ and the specific form of $\vartheta(\mathcal{U})$ as a measurable function on $(\Omega, \mathcal{F}, \mathbb{P})$ for a given $\mathcal{U}$. However, the distribution $\mathbb{P} \circ(\mathcal{U}, \vartheta(\mathcal{U}))^{-1}$ on $\mathcal{A}^{+} \times \mathcal{A}^{+}$is unambiguously fixed by (1). See the Appendix for an explicit choice of $\left(\Omega_{2}, \mathcal{F}_{2}, \mathbb{P}_{2}\right)$ and $\vartheta$, showing that a random substitution always exists.

Let us discuss some special cases. First, suppose $\Omega_{1}=\{a\}, \mathbb{P}_{1}=\delta_{a}$ and $\mathcal{U}(a)=a$ is the trivial random variable equal to $a \in \mathcal{A}$. In that case, we write $\vartheta(a):=\vartheta(\mathcal{U})$ and we find $\mathbb{P}[\vartheta(a)=v]=\mathbb{P}[\vartheta(a)=v \mid \mathcal{U}=a]=P(a, v)=P_{a}^{\vartheta}(v)$ for all $v \in \mathcal{A}^{+}$. That is, the distribution of $\vartheta(a)$ on $\mathcal{A}^{+}$is given by $P_{a}^{\vartheta}$. If $\operatorname{supp} P_{a}^{\vartheta}=\left\{v^{1}, \ldots, v^{m}\right\}$ and $P_{a}^{\vartheta}\left(v^{j}\right)=p_{j}$ for $j \in\{1, \ldots, m\}$, we represent this distribution in the following form

$$
\vartheta(a)= \begin{cases}v^{1}, & \text { with probability } p_{1} \\ \vdots & \\ v^{m} & \text { with probability } p_{m}\end{cases}
$$


compare also the notation in [28]. This is consistent with the observation $\mathbb{P}\left[\vartheta(a)=v^{j}\right]=p_{j}$, for $j \in\{1, \ldots, m\}$, by the above calculation. Note that this holds also in the slightly more general situation that the distribution of $\mathcal{U}$ is given by $\delta_{a}$.

Example 2.9. Let $\mathcal{A}=\{a, b\}$. The random Fibonacci substitution rule is given by the two probability distributions $P_{a}^{\vartheta}$ and $P_{b}^{\vartheta}$, with $P_{a}^{\vartheta}(a b)=p_{1}, P_{a}^{\vartheta}(b a)=p_{2}=1-p_{1}$ and $P_{b}^{\vartheta}(a)=1$. Alternatively, in line with the previous discussion,

$$
\vartheta: b \mapsto a, \quad a \mapsto \begin{cases}a b, & \text { with probability } p_{1}, \\ b a, & \text { with probability } p_{2},\end{cases}
$$

for the distribution of a corresponding random substitution acting on letters. Note that this contains the same information as the random substitution rule. It therefore suffices in order to determine the transition kernel $P$ and hence the common distribution of $(\mathcal{U}, \vartheta(\mathcal{U})$ ) for an arbitrary random word $\mathcal{U}$. We call a random substitution with distributions fixed by (2) random Fibonacci substitution. Glossing over the ambiguity in the choice of the underlying probability space, we say that (2) determines the random substitution $\vartheta$.

Let us fix an initial random word $\mathcal{U} \in \mathcal{A}^{+}$and construct a sequence of random words $\left(\vartheta^{n}(\mathcal{U})\right)_{n \in \mathbb{N}}$ by iterating the random substitution $\vartheta$. For $j \in \mathbb{N}, \vartheta^{j}(\mathcal{U})$ is a random word on a probability space $\left(X_{k=1}^{j+1} \Omega_{k}, \bigotimes_{k=1}^{j+1} \mathcal{F}_{k}, \bigotimes_{k=1}^{j+1} \mathbb{P}_{k}\right)$. We can thus embed all $\vartheta^{j}(\mathcal{U})$ as functions on a probability space $\left(\Omega_{\mathcal{U}}, \mathcal{F}_{\mathcal{U}}, \mathbb{P}_{\mathcal{U}}\right)=\left(X_{k=1}^{\infty} \Omega_{k}, \bigotimes_{k=1}^{\infty} \mathcal{F}_{k}, \bigotimes_{k=1}^{\infty} \mathbb{P}_{k}\right)$, depending only on the first $j+1$ coordinates. By definition, $\left(\vartheta^{n}(\mathcal{U})\right)_{n \in \mathbb{N}}$ forms a stationary Markov chain on $\left(\Omega_{\mathcal{U}}, \mathcal{F}_{\mathcal{U}}, \mathbb{P}_{\mathcal{U}}\right)$ with transition probabilities

$$
\mathbb{P}_{\mathcal{U}}\left[\vartheta^{n+1}(\mathcal{U})=w \mid \vartheta^{n}(\mathcal{U})=v\right]=P(v, w),
$$

for all $n \in \mathbb{N}_{0}$ and $v, w \in \mathcal{A}^{+}$, where we have used $\vartheta^{0}(\mathcal{U})=\mathcal{U}$ as a convention. Inductively, we find $\mathbb{P}_{\mathcal{U}}\left[\vartheta^{n+k}(\mathcal{U})=w \mid \vartheta^{n}(\mathcal{U})=v\right]=P^{k}(v, w)$, for all $n, k \in \mathbb{N}$. Since $P^{k}(v, w)=\mathbb{P}_{v}\left[\vartheta^{k}(v)=\right.$ $w]$, we obtain

$$
\begin{aligned}
\mathbb{P}_{\mathcal{U}}\left[\vartheta^{n+k}(\mathcal{U})=w\right] & =\sum_{v \in \mathcal{A}^{+}} \mathbb{P}_{\mathcal{U}}\left[\vartheta^{n+k}(\mathcal{U})=w \mid \vartheta^{n}(\mathcal{U})=v\right] \mathbb{P}_{\mathcal{U}}\left[\vartheta^{n}(\mathcal{U})=v\right] \\
& =\sum_{v \in \mathcal{A}^{+}} \mathbb{P}_{\mathcal{U}}\left[\vartheta^{n}(\mathcal{U})=v\right] \mathbb{P}_{v}\left[\vartheta^{k}(v)=w\right]
\end{aligned}
$$

Next, assume that we start from a deterministic word $\mathcal{U} \equiv u$, for some $u=u_{1} \ldots u_{m} \in \mathcal{A}^{+}$. First, observe that $P(u, v)=\left(\bigotimes_{j=1}^{m} \mathbb{P}_{u_{j}}\right)\left[\vartheta\left(u_{1}\right) \cdots \vartheta\left(u_{m}\right)=v\right]$, for all $v \in \mathcal{A}^{+}$, compare Remark 2.6. Consider the Markov chain $\left(\vartheta^{n}(u)\right)_{n \in \mathbb{N}}$ on $\left(\Omega_{u}, \mathcal{F}_{u}, \mathbb{P}_{u}\right)$. For each $j \in\{1, \ldots, m\}$ we also have a Markov chain $\left(\vartheta^{n}\left(u_{j}\right)\right)_{n \in \mathbb{N}}$. It is possible to realise each $\vartheta^{n}\left(u_{j}\right)$ as a function on $\left(\Omega_{u}, \mathcal{F}_{u}, \mathbb{P}_{u}\right)$ in such a way that $\vartheta^{n}(u)(\omega)=\vartheta^{n}\left(u_{1}\right)(\omega) \cdots \vartheta^{n}\left(u_{m}\right)(\omega)$ while preserving the defining relation $\mathbb{P}_{u}\left[\vartheta^{n+1}\left(u_{j}\right)=w \mid \vartheta^{n}\left(u_{j}\right)=v\right]=P(v, w)$ and independence of $\vartheta^{n}\left(u_{j}\right)$ and $\vartheta^{n}\left(u_{k}\right)$ for $j \neq k$. We refer to the Appendix for details. In particular,

$$
\mathbb{P}_{u}\left[\vartheta^{n}(u)=v\right]=\mathbb{P}_{u}\left[\vartheta^{n}\left(u_{1}\right) \cdots \vartheta^{n}\left(u_{m}\right)=v\right],
$$

for all $v \in \mathcal{A}^{+}$. This can also be verified using properties of the transition kernel $P$ only. 
Remark 2.10. There is some redundancy in notations like $\mathbb{P}_{\mathcal{U}}\left[\vartheta^{n}(\mathcal{U})=v\right]$, as the initial random word $\mathcal{U}$ is encoded both as an argument of $\vartheta^{n}$ and as a subscript of $\mathbb{P}$. In such situations, we will therefore often write $\mathbb{P}$ instead of $\mathbb{P}_{\mathcal{U}}$. The same holds for the expectation value $\mathbb{E}=\mathbb{E}_{\mathcal{U}}$.

Remark 2.11. Suppose $\vartheta$ is a random substitution on the alphabet $\mathcal{A}$. Let us define a set-valued map on $\mathcal{A}$ via $\hat{\vartheta}(a):=\operatorname{supp}\left(\mathbb{P} \circ \vartheta(a)^{-1}\right) \subset \mathcal{A}^{+}$. In words, $\hat{\vartheta}(a)$ consists of the set of possible realisations of $\vartheta(a)$. We can extend $\hat{\vartheta}$ to words via

$$
\hat{\vartheta}\left(u_{1} \cdots u_{m}\right)=\left\{v^{1} \cdots v^{m} \mid v^{j} \in \hat{\vartheta}\left(u_{j}\right) \text { for all } j \in\{1, \ldots, m\}\right\}
$$

and to sets of words as $\hat{\vartheta}(A)=\cup_{u \in A} \hat{\vartheta}(u)$. This is what is called a 'random substitution' in [10]. It is a straightforward exercise to verify inductively that $\hat{\vartheta}^{n}(u)=\operatorname{supp}\left(\mathbb{P} \circ \vartheta^{n}(u)^{-1}\right)$ for all $n \in \mathbb{N}$, so $v \in \hat{\vartheta}^{n}(u)$ if and only if $\mathbb{P}\left[\vartheta^{n}(u)=v\right]>0$. We refer to $\hat{\vartheta}$ as a multi-valued substitution in our present context.

Example 2.12. Recall the random Fibonacci substitution $\vartheta$ from Example 2.9. The distribution of $\vartheta^{2}(b)=\vartheta(\vartheta(b))=\vartheta(a)$ is immediate. The probability that $\vartheta^{2}(a)=a b a$ can be determined to be

$$
\begin{aligned}
\mathbb{P}\left[\vartheta^{2}(a)=a b a\right] & =\sum_{v \in \mathcal{A}^{+}} \mathbb{P}[\vartheta(a)=v] \mathbb{P}[\vartheta(v)=a b a] \\
& =\mathbb{P}[\vartheta(a)=a b] \mathbb{P}[\vartheta(a) \vartheta(b)=a b a]+\mathbb{P}[\vartheta(a)=b a] \mathbb{P}[\vartheta(b) \vartheta(a)=a b a] \\
& =p_{1}^{2}+p_{2}^{2}
\end{aligned}
$$

Note that there are two different realisations $(a b, a b a)$ and $(b a, a b a)$ of $\left(\vartheta(a), \vartheta^{2}(a)\right)$, contributing to the event $\left[\vartheta^{2}(a)=a b a\right]$. The full distribution of $\vartheta^{2}(a)$ is given by

$$
\vartheta^{2}(a)= \begin{cases}a a b, & \text { with probability } p_{2} p_{1}, \\ a b a, & \text { with probability } p_{1}^{2}+p_{2}^{2}, \\ b a a, & \text { with probability } p_{1} p_{2} .\end{cases}
$$

Example 2.13. Consider the random substitution

$$
\vartheta: a \mapsto\left\{\begin{array} { l l } 
{ b , } & { \text { with probability } p _ { 1 } , } \\
{ b a , } & { \text { with probability } p _ { 2 } , }
\end{array} \quad b \mapsto \left\{\begin{array}{ll}
b, & \text { with probability } q_{1}, \\
a b, & \text { with probability } q_{2} .
\end{array}\right.\right.
$$

Then, the event $[\vartheta(a b)=b a b]$ can be realised either via $\vartheta(a)=b a, \vartheta(b)=b$ or via $\vartheta(a)=b$, $\vartheta(b)=a b$. For the transition kernel $P$, we therefore find

$$
\begin{aligned}
P(a b, b a b) & =P_{a}^{\vartheta}(b a) P_{b}^{\vartheta}(b)+P_{a}^{\vartheta}(b) P_{b}^{\vartheta}(a b) \\
& =\mathbb{P}[\vartheta(a)=b a] \mathbb{P}[\vartheta(b)=b]+\mathbb{P}[\vartheta(a)=b] \mathbb{P}[\vartheta(b)=a b]=p_{2} q_{1}+p_{1} q_{2} .
\end{aligned}
$$

This is one of the cases where the pair $(\vartheta(a), \vartheta(b))$ contains more information than their concatenation $\vartheta(a) \vartheta(b)=\vartheta(a b)$.

In the same spirit as for deterministic substitutions, we can define a substitution matrix which captures some information about the Abelianisation of the random substitution process. 
Definition 2.14. The mean substitution matrix $M_{\vartheta}$ of a random substitution $\vartheta$ is defined via $\left(M_{\vartheta}\right)_{a b}=\mathbb{E}\left[|\vartheta(b)|_{a}\right]=\mathbb{E}\left[\Phi(\vartheta(b))_{a}\right]$ for all $a, b \in \mathcal{A}$. A random substitution $\vartheta$ is called primitive if $M_{\vartheta}$ is a primitive matrix. In this case, it is called expanding if $\mathbb{P}[|\vartheta(a)|>1]>0$ for some $a \in \mathcal{A}$. For a primitive random substitution, we denote by $\lambda$ the Perron-Frobenius (PF) eigenvalue of $M_{\vartheta}$ and by $R$ and $L$ the corresponding right and left eigenvectors, respectively. The normalisation of these vectors is chosen such that $\|R\|_{1}=1$ and $L \cdot R=1$.

Remark 2.15. Unlike in the deterministic setting, a primitive random substitution need not be expanding. An easy counterexample is given by

$$
\vartheta: a, b \mapsto\left\{\begin{array}{ll}
a, & \text { with probability } p_{1}, \\
b, & \text { with probability } p_{2},
\end{array} \quad M_{\vartheta}=\left(\begin{array}{ll}
p_{1} & p_{1} \\
p_{2} & p_{2}
\end{array}\right) .\right.
$$

We can construct from a random substitution the set of legal words $\mathcal{L}_{\vartheta}$ and the symbolic hull $\mathbb{X}_{\vartheta}$, much as in the deterministic case, compare [20], [28].

Definition 2.16. The language of a random substitution $\vartheta$ on $\mathcal{A}$ is defined as

$$
\mathcal{L}_{\vartheta}=\left\{u \in \mathcal{A}^{+}: u \triangleleft v, \mathbb{P}\left[\vartheta^{n}(a)=v\right]>0, \text { for some } n \in \mathbb{N}, a \in \mathcal{A}\right\} .
$$

The set of $\left(\vartheta\right.$-)legal words of length $\ell$ is given by $\mathcal{L}_{\vartheta}^{\ell}=\left\{u \in \mathcal{L}_{\vartheta}:|u|=\ell\right\}$.

The definition of $\mathcal{L}_{\vartheta}$ is independent of the probability vectors (as long as none of their entries is 0 ), making it a purely combinatorial object. In fact, it can already be constructed from the multi-valued substitution $\hat{\vartheta}$ pertaining to $\vartheta$, compare Remark 2.11. The same holds for the subshift that we can construct from this language.

Definition 2.17. For a random substitution $\vartheta$ on $\mathcal{A}$, we define the random substitution subshift (RS-subshift) or stochastic hull as

$$
\mathbb{X}_{\vartheta}=\left\{x \in \mathcal{A}^{\mathbb{Z}}: x_{[k, m]} \in \mathcal{L}_{\vartheta} \text { for all } k \leqslant m\right\} .
$$

A probabilistic structure on $\mathbb{X}_{\vartheta}$ will be obtained by a convenient choice of a measure on this subshift in Section 5.

\section{WORD FREQUENCIES, CONVERGENCE IN EXPECTATION}

Many of the results in this section essentially go back to [13]. However, we do not assume the existence of a letter $b \in \mathcal{A}$ and a realisation $\omega \in \Omega_{b}$ such that $\vartheta(b)(\omega)$ starts with $b$, which is taken as a standing assumption in [13]. Also note that the terminology in [13] is slightly different in so far as properties that hold under taking expectation are often called 'almost sure'.

An important tool for determining letter frequencies of a deterministic substitution is its corresponding induced substitution [25, Ch. 5.4.1]. Before we continue, we need to extend this notion to the case of expanding random substitutions. For an arbitrary $\ell \in \mathbb{N}$, let $\mathcal{A}_{\ell}:=\mathcal{L}_{\vartheta}^{\ell}$ be the alphabet consisting of letters that are $\vartheta$-legal words of length $\ell$. We define a subset of consistent right collared words as

$$
\mathcal{D}_{\ell}=\left\{V \in \mathcal{A}_{\ell}^{+}: V=\left(v_{[k, k+\ell-1]}\right)_{1 \leqslant k \leqslant m} \text { for some } m \in \mathbb{N}, v \in \mathcal{A}^{+}\right\} .
$$


For $v \in \mathcal{A}^{+}$, let us assume that $\vartheta^{n}\left(v_{j}\right)$ is a random variable on $\left(\Omega_{v}, \mathcal{F}_{v}, \mathbb{P}_{v}\right)$ for all $1 \leqslant j \leqslant|v|$, compare the Appendix. In that case, we set $\vartheta^{n}\left(v_{k} \cdots v_{m}\right)(\omega)=\vartheta^{n}\left(v_{k}\right)(\omega) \cdots \vartheta^{n}\left(v_{m}\right)(\omega)$ for $1 \leqslant k \leqslant m \leqslant|v|$ and $\omega \in \Omega_{v}$.

Definition 3.1. Let $\vartheta$ be an expanding random substitution, $\ell \in \mathbb{N}$ and $V=V_{1} \cdots V_{m}=$ $\left(v_{[k, k+\ell-1]}\right)_{1 \leqslant k \leqslant m} \in \mathcal{D}_{\ell}$ with $v=v_{1} \cdots v_{m+\ell-1}$. For $n \in \mathbb{N}$, the ( $\left.\ell-\right)$ induced substitution of $\vartheta^{n}(v)$ is a random word $\vartheta_{\ell}^{n}(V)$ on the probability space $\left(\Omega_{v}, \mathcal{F}_{v}, \mathbb{P}_{v}\right)$, with values in $\mathcal{D}_{\ell}$. It is defined as

$$
\vartheta_{\ell}^{n}(V)(\omega)=\left(\vartheta^{n}(v)(\omega)_{[k, k+\ell-1]}\right)_{1 \leqslant k \leqslant\left|\vartheta^{n}\left(v_{1} \cdots v_{m}\right)(\omega)\right|} .
$$

The pairs $\left(\vartheta^{n}(v), \vartheta^{n}\left(v_{1} \cdots v_{m}\right)\right)_{n \in \mathbb{N}}$ form a stationary Markov chain by definition. Since each $\vartheta_{\ell}^{n}(V)$ is a function of $\left(\vartheta^{n}(v), \vartheta^{n}\left(v_{1} \cdots v_{m}\right)\right)$, the same holds for the sequence $\left(\vartheta_{\ell}^{n}(V)\right)_{n \in \mathbb{N}}$. We have the identity $\vartheta_{\ell}^{n}(V)=\vartheta_{\ell}^{n}\left(V_{1}\right) \cdots \vartheta_{\ell}^{n}\left(V_{m}\right)$, with

$$
\vartheta_{\ell}^{n}\left(V_{j}\right)(\omega)=\left(\vartheta^{n}\left(V_{j}\right)(\omega)_{[k, k+\ell-1]}\right)_{1 \leqslant k \leqslant\left|\vartheta^{n}\left(v_{j}\right)(\omega)\right|}
$$

for all $n \in \mathbb{N}, j \in\{1, \ldots, m\}$ and $\omega \in \Omega_{u}$.

Remark 3.2. It is possible to define an induced substitution $\vartheta_{\ell}$ also as a map between

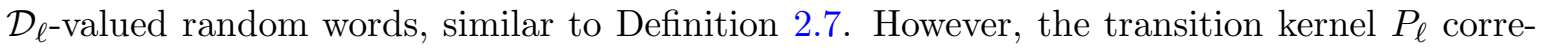
sponding to $\vartheta_{\ell}$ does not have the same product structure as $P$. This corresponds to the fact that for $V=V_{1} \cdots V_{m}$, the random words $\vartheta_{\ell}\left(V_{1}\right), \ldots, \vartheta_{\ell}\left(V_{m}\right)$ are not independent. That is because e.g. the words $V_{1}$ and $V_{2}$ have some overlap which, by force, is mapped to the same word under $\vartheta$ for any realisation. The Markov chain $\left(\vartheta_{\ell}^{n}(V)\right)_{n \in \mathbb{N}}$ forms an M-system in the sense of Peyrière [23].

We again define an Abelianisation map $\Phi_{\ell}: \mathcal{D}_{\ell} \rightarrow \mathbb{N}_{0}^{\text {card } \mathcal{A}_{\ell}}$ via $\Phi_{\ell}(V)_{u}=|V|_{u}$ for all $V \in \mathcal{D}_{\ell}$ and $u \in \mathcal{A}_{\ell}$. The mean substitution matrix $M_{\vartheta_{\ell}}$ is given by $\left(M_{\vartheta_{\ell}}\right)_{u v}=\mathbb{E}\left[\left|\vartheta_{\ell}(v)\right|_{u}\right]=$ $\mathbb{E} \Phi_{\ell}\left(\vartheta_{\ell}(v)\right)_{u}$ for all $u, v \in \mathcal{A}_{\ell}$. Denote by $L^{(\ell)}$ and $R^{(\ell)}$ the corresponding left and right eigenvectors, normalized as $L^{(\ell)} \cdot R^{(\ell)}=1$ and $\left\|R^{(\ell)}\right\|_{1}=1$. Note that for $\ell=1$, we have $\Phi_{\ell}=\Phi$ and $M_{\vartheta_{\ell}}=M_{\vartheta}$, compare Definition 2.14.

Proposition 3.3. Let $\vartheta$ be a random substitution, $\ell \in \mathbb{N}$ and $V=V_{1} \cdots V_{m}=\left(v_{[k, k+\ell-1]}\right)_{1 \leqslant k \leqslant m}$ with $v=v_{1} \cdots v_{m+\ell-1} \in \mathcal{A}^{+}$. If $\ell>1$, suppose further that $\vartheta$ is expanding. Then, for all $n \in \mathbb{N}$, we have the following conditional expectation,

$$
\mathbb{E}\left[\Phi_{\ell}\left(\vartheta_{\ell}^{n}(V)\right) \mid \Phi_{\ell}\left(\vartheta_{\ell}^{n-1}(V)\right)\right]=M_{\vartheta_{\ell}} \Phi_{\ell}\left(\vartheta_{\ell}^{n-1}(V)\right) .
$$

In particular, $\mathbb{E}\left[\Phi_{\ell}\left(\vartheta_{\ell}^{n}(V)\right)\right]=M_{\vartheta_{\ell}} \mathbb{E}\left[\Phi_{\ell}\left(\vartheta_{\ell}^{n-1}(V)\right)\right]=M_{\vartheta_{\ell}}^{n} \Phi_{\ell}(V)$.

Proof. Because $\left(\vartheta_{\ell}^{n}(V)\right)_{n \in \mathbb{N}}$ is a stationary process, it suffices to show

$$
\mathbb{E}\left[\Phi_{\ell}\left(\vartheta_{\ell}(V)\right)\right]=\mathbb{E}\left[\Phi_{\ell}\left(\vartheta_{\ell}(V)\right) \mid \Phi_{\ell}(V)\right]=M_{\vartheta_{\ell}} \Phi_{\ell}(V) .
$$

For $u \in \mathcal{A}_{\ell}$, we can conclude from the pointwise identity $\left|\vartheta_{\ell}(V)(\omega)\right|_{u}=\sum_{i=1}^{m}\left|\vartheta_{\ell}\left(V_{i}\right)(\omega)\right|_{u}$ the corresponding relation under taking expectations,

$$
\mathbb{E}\left|\vartheta_{\ell}(V)\right|_{u}=\sum_{i=1}^{m} \mathbb{E}\left|\vartheta_{\ell}\left(V_{i}\right)\right|_{u}=\sum_{v \in \mathcal{A}_{\ell}}\left(M_{\vartheta_{\ell}}\right)_{u v} \Phi_{\ell}(V)_{v}=\left(M_{\vartheta_{\ell}} \Phi_{\ell}(V)\right)_{u} .
$$

Since $\mathbb{E} \Phi_{\ell}\left(\vartheta_{\ell}(V)\right)_{u}=\mathbb{E}\left|\vartheta_{\ell}(V)\right|_{u}$, this proves the claim. 
For $\ell=1$, the induced substitution $\vartheta_{1}$ coincides with the original substitution $\vartheta$. This yields the following alternative characterisation of primitive substitutions, compare [28, Rem. 6] and [20, Rem. 2.13].

Corollary 3.4. Let $\vartheta$ be a random substitution rule on $\mathcal{A}$. Then, $\vartheta$ is primitive if and only if there exists a number $k \in \mathbb{N}$ such that, for all $a, b \in \mathcal{A}$, we have $\mathbb{P}\left[a \triangleleft \vartheta^{k}(b)\right]>0$.

Proof. From Proposition 3.3, we know $\mathbb{E} \Phi\left(\vartheta^{k}(b)\right)_{a}=\left(M_{\vartheta}^{k} \Phi(b)\right)_{a}=\left(M_{\vartheta}^{k}\right)_{a b}$. Therefore,

$$
\left(M_{\vartheta}^{k}\right)_{a b}>0 \Longleftrightarrow \mathbb{E} \Phi\left(\vartheta^{k}(b)\right)_{a}>0 \Longleftrightarrow \mathbb{P}\left[\left|\vartheta^{k}(b)\right|_{a}>0\right]>0 .
$$

It was shown in [28, Prop. 26] that $M_{\vartheta}$ is primitive if $M_{\vartheta}$ is primitive and in [13, Lem. 2.4.9] that the $\mathrm{PF}$ eigenvalues of both matrices coincide. The following result is inspired by [13, Lem. 2.4.5, Lem. 2.4.6, Cor. 2.4.8]. Note that our results differs from the one given in the proof of [13, Lem. 2.4.5] by a factor of $L_{u}^{(\ell)}$ as we fixed the norm of the right eigenvector to be 1 .

Lemma 3.5. Let $\vartheta$ be an expanding primitive random substitution on the finite alphabet $\mathcal{A}$. For any $\ell \in \mathbb{N}$, let $\vartheta_{\ell}$ be the induced substitution with mean substitution matrix $M_{\vartheta_{\ell}}$, $P F$ eigenvalue $\lambda$ and right and left PF-eigenvector $R^{(\ell)}$ and $L^{(\ell)}$, normalised as above. Then, we have the following convergence results:

$$
\begin{aligned}
& \lim _{n \rightarrow \infty} \frac{\mathbb{E} \Phi_{\ell}\left(\vartheta_{\ell}^{n}(u)\right)}{\lambda^{n}}=L_{u}^{(\ell)} R^{(\ell)}, \\
& \lim _{n \rightarrow \infty} \frac{\mathbb{E}\left|\vartheta_{\ell}^{n+1}(u)\right|}{\mathbb{E}\left|\vartheta_{\ell}^{n}(u)\right|}=\lambda, \\
& \lim _{n \rightarrow \infty} \frac{\mathbb{E} \Phi_{\ell}\left(\vartheta_{\ell}^{n}(u)\right)}{\mathbb{E}\left|\vartheta_{\ell}^{n}(u)\right|}=R^{(\ell)},
\end{aligned}
$$

for all $u \in \mathcal{A}_{\ell}$. The same statement is still true for non-expanding $\vartheta$ if $\ell=1$.

Proof. Note that $\left\|\Phi_{\ell}\left(\vartheta_{\ell}^{n}(u)\right)\right\|_{1}=\left|\vartheta_{\ell}^{n}(u)\right|$ and the expectation value is a linear operator, so the second and the third relations follow immediately from the first, observing the identity $\frac{1}{\lambda^{n}} \mathbb{E}\left|\vartheta_{\ell}^{n}(u)\right| \stackrel{n \rightarrow \infty}{\longrightarrow} L_{u}^{(\ell)} \neq 0$. It thus remains to show Eq. (3).

Using Proposition 3.3, we find $\mathbb{E} \Phi_{\ell}\left(\vartheta_{\ell}^{n}(u)\right)=M_{\vartheta_{\ell}}^{n} \Phi_{\ell}(u)$ and thus, by an application of the PF theorem,

$$
\frac{\mathbb{E} \Phi_{\ell}\left(\vartheta_{\ell}^{n}(u)\right)}{\lambda^{n}}=\frac{1}{\lambda^{n}} M_{\vartheta_{\ell}}^{n} \Phi_{\ell}(u) \stackrel{n \rightarrow \infty}{\longrightarrow} R^{(\ell)}\left(L^{(\ell)} \cdot e_{u}\right)=R^{(\ell)} L_{u}^{(\ell)} .
$$

In the next result, we establish an exponentially decaying bound for the probability that the length of $\vartheta^{n}(a)$ grows sublinearly. This will be a convenient tool in later constructions.

Proposition 3.6. If $\vartheta$ is an expanding primitive substitution, there are constants $C, K>0$ such that, for all $a \in \mathcal{A}$ and large enough $n$,

$$
\mathbb{P}\left[\left|\vartheta^{n}(a)\right|<K n\right] \leqslant \mathrm{e}^{-C n} .
$$


In particular,

$$
\lim _{n \rightarrow \infty}\left|\vartheta^{n}(a)\right|=\infty
$$

almost surely for all $a \in \mathcal{A}$.

Proof. The second claim is an immediate consequence of the first one by an application of the Borel-Cantelli lemma.

Let $b \in A$ and $u$ be a word such that $|u| \geqslant 2$ and $\mathbb{P}[\vartheta(b)(\omega)=u]>0$. From the primitivity of $\vartheta$, we deduce that there is $\widetilde{N} \in \mathbb{N}$ such that, for all $a \in \mathcal{A}$, we have $b \triangleleft \vartheta^{\widetilde{N}}(a)$, and consequently $u \triangleleft \vartheta^{\widetilde{N}+1}(a)$ with positive probability $\widetilde{q}$. Set $N:=\widetilde{N}+1$. It follows

$$
\mathbb{P}\left[\left|\vartheta^{N}(a)\right| \geqslant 2\right] \geqslant \widetilde{q},
$$

hence,

$$
\mathbb{P}\left[\left|\vartheta^{N}(a)\right|=1\right] \leqslant(1-\widetilde{q})=: q<1 .
$$

Since this holds for all $a \in \mathcal{A}$, we obtain for every $v \in \mathcal{A}^{+}, \mathbb{P}\left[\left|\vartheta^{N}(v)\right|=|v|\right] \leqslant q^{|v|} \leqslant q$. By stationarity, this yields for every $k \in \mathbb{N}$,

$$
\mathbb{P}\left[\left|\vartheta^{N k}(a)\right|=\left|\vartheta^{N(k-1)}(a)\right|\right] \leqslant q .
$$

For every $n \in \mathbb{N}$ and $\omega \in \Omega_{a}$, let us define

$$
J_{n}(\omega)=\left\{j \in\{1, \ldots, n\}:\left|\vartheta^{N j}(a)(\omega)\right|=\left|\vartheta^{N(j-1)}(a)(\omega)\right|\right\} .
$$

Combining Eq. (6) with the Markov property, we obtain for every $J \subset\{1, \ldots, n\}$,

$$
\mathbb{P}\left[J \subset J_{n}\right] \leqslant q^{\operatorname{card}(J)} .
$$

Suppose $0<K<1$. Then, $\left|\vartheta^{N n}(a)(\omega)\right| \leqslant K n$ implies card $\left(J_{n}(\omega)\right) \geqslant n-\lceil K n\rceil$. Simple combinatorial arguments yield

$$
\begin{aligned}
\mathbb{P}\left[\left|\vartheta^{N n}(a)\right| \leqslant K n\right] & \leqslant \mathbb{P}\left[\operatorname{card} J_{n} \geqslant n-\lceil K n\rceil\right]=\sum_{k=n-\lceil K n\rceil}^{n} \mathbb{P}\left[\operatorname{card} J_{n}=k\right] \\
& \leqslant \sum_{k=n-\lceil K n\rceil}^{n}\left(\begin{array}{l}
n \\
k
\end{array}\right) q^{k} \leqslant(\lceil K n\rceil+1)\left(\begin{array}{c}
n \\
\lceil K n\rceil
\end{array}\right) q^{n-\lceil K n\rceil} .
\end{aligned}
$$

Using Stirling's formula, we find

$$
\liminf _{n \rightarrow \infty} \frac{1}{n} \log \left(\begin{array}{c}
n \\
\lceil K n\rceil
\end{array}\right)=-K \log (K)-(1-K) \log (1-K)
$$

and thus

$$
\begin{aligned}
\liminf _{n \rightarrow \infty} \frac{1}{n} \log \left(\mathbb{P}\left[\left|\vartheta^{N n}(a)\right| \leqslant K n\right]\right) & \leqslant(1-K) \log (q)-K \log (K)-(1-K) \log (1-K) \\
& \stackrel{K \rightarrow 0}{\longrightarrow} \log (q)<0 .
\end{aligned}
$$

Thereby, we can find $\widetilde{K}, \widetilde{C}>0$ such that

$$
\mathbb{P}\left[\left|\vartheta^{N n}(a)\right| \leqslant \widetilde{K} n\right] \leqslant \mathrm{e}^{-\widetilde{C} n}
$$


for large enough $n$. Setting $C=\frac{\widetilde{C}}{2 N}$ and $K=\frac{\widetilde{K}}{2 N}$, the assertion follows by a straightforward interpolation argument.

Corollary 3.7. A primitive random substitution $\vartheta$ is expanding if and only if the corresponding PF-eigenvalue $\lambda$ fulfils $\lambda>1$.

Proof. We will use the assertion of Lemma 3.5 for the case $\ell=1$, such that it is still true for non-expanding substitutions. Therefore, $\lambda>1$ is equivalent to $\mathbb{E}\left|\vartheta^{n}(a)\right| \rightarrow \infty$ as $n \rightarrow \infty$. So, $\lambda>1$ clearly implies that $\vartheta$ is an expanding substitution. On the other hand, if $\vartheta$ is expanding, $\left|\vartheta^{n}(a)(\omega)\right| \rightarrow \infty$, as $n \rightarrow \infty$, for all $\omega \in B$ with $\mathbb{P}(B)=1$, by Proposition 3.6. Therefore,

$$
\liminf _{n \rightarrow \infty} \mathbb{E}\left|\vartheta^{n}(a)\right|=\liminf _{n \rightarrow \infty} \int_{B}\left|\vartheta^{n}(a)(\omega)\right| \mathrm{d} \mathbb{P}(\omega) \geqslant \int_{B} \liminf _{n \rightarrow \infty}\left|\vartheta^{n}(a)(\omega)\right| \mathrm{d} \mathbb{P}(\omega)=\infty,
$$

by Fatou's Lemma. Thus, $\lim _{n \rightarrow \infty} \mathbb{E}\left|\vartheta^{n}(a)\right|=\infty$ for all $a \in \mathcal{A}$, implying $\lambda>1$.

We finally conclude with the following result — compare the proof of [13, Thm. 2.4.10].

Proposition 3.8. Let $\vartheta$ be an expanding, primitive random substitution on $\mathcal{A}$. For any $\ell \in \mathbb{N}$, let $\vartheta_{\ell}$ be the induced substitution with mean substitution matrix $M_{\vartheta_{\ell}}$ and right normalised PF eigenvector $R^{(\ell)}$. Then, for any $v \in \mathcal{L}_{\vartheta}^{\ell}$ and $a \in \mathcal{A}$,

$$
\lim _{n \rightarrow \infty} \frac{\mathbb{E}\left|\vartheta^{n}(a)\right|_{v}}{\mathbb{E}\left|\vartheta^{n}(a)\right|}=R_{v}^{(\ell)}
$$

Furthermore, for any $u \in \mathcal{L}_{\vartheta}^{\ell}$ with $u_{1}=a$,

$$
L_{u}^{(\ell)}=\lim _{n \rightarrow \infty} \frac{\mathbb{E}\left|\vartheta_{\ell}^{n}(u)\right|}{\lambda^{n}}=\lim _{n \rightarrow \infty} \frac{\mathbb{E}\left|\vartheta^{n}(a)\right|}{\lambda^{n}}=L_{a} .
$$

Proof. Let $\ell \in \mathbb{N}, a \in \mathcal{A}$ and $v \in \mathcal{L}_{\vartheta}^{\ell}$. Since $\vartheta$ is expanding and primitive, there are words of arbitrary length containing $a$ - compare Corollary 3.4. Choose any legal word of length $\ell$, $u=a a_{2} \cdots a_{\ell} \in \mathcal{L}_{\vartheta}^{\ell}$, starting with the letter $a$. From the definition of the induced substitution, we find $\left|\vartheta_{\ell}^{n}(u)(\omega)\right|=\left|\vartheta^{n}(a)(\omega)\right|$, for all $\omega \in \Omega$. This also implies that $\mathbb{E}\left|\vartheta_{\ell}^{n}(u)\right|=\mathbb{E}\left|\vartheta^{n}(a)\right|$, for every $n \in \mathbb{N}$, yielding the second identity when combined with Eq. (3).

Furthermore, we have $\left|\vartheta_{\ell}^{n}(u)(\omega)\right|_{v}=\left|\vartheta^{n}(a)(\omega)\right|_{v}+\mathcal{O}(\ell)$. This is because the last $\ell-1$ 'letters' $\left(\in \mathcal{L}_{\vartheta}^{\ell}\right)$ of $\vartheta_{\ell}^{n}(u)(\omega)$ are not entirely included in $\vartheta^{n}(a)(\omega)$, whereas all others comprise the subwords of $\vartheta^{n}(a)(\omega)$. Thus, $\mathbb{E}\left|\vartheta_{\ell}^{n}(u)\right|_{v}=\mathbb{E}\left|\vartheta^{n}(a)\right|_{v}+\mathcal{O}(\ell)$. Since $\vartheta$ is expanding, it is $\lambda>1$ and so the error term $\mathcal{O}(\ell)$, when divided by $\mathbb{E}\left|\vartheta^{n}(a)\right|$, gets arbitrarily small, as $n \rightarrow \infty$. Consequently, writing $\left|\vartheta_{\ell}^{n}(u)\right|_{v}=\Phi_{\ell}\left(\vartheta_{\ell}^{n}(u)\right)_{v}$,

$$
\lim _{n \rightarrow \infty} \frac{\mathbb{E}\left|\vartheta^{n}(a)\right|_{v}}{\mathbb{E}\left|\vartheta^{n}(a)\right|}=\lim _{n \rightarrow \infty} \frac{\mathbb{E}\left|\vartheta_{\ell}^{n}(u)\right|_{v}}{\mathbb{E}\left|\vartheta_{\ell}^{n}(u)\right|}=R_{v}^{(\ell)},
$$

by an application of the third identity in Lemma 3.5 .

For the rest of this paper, we set $\vartheta$ to be an expanding primitive random substitution on some finite alphabet $\mathcal{A}$ with $m$ letters. This allows us to use the powerful machinery derived from PF theory which we developed in this chapter. 


\section{WORD FREQUENCIES IN INFLATION WORDS}

The word frequency of some $v \in \mathcal{L}_{\vartheta}^{\ell}$ within a (generally larger) word $w \in \mathcal{L}_{\vartheta}$ will be denoted by $\nu_{v}(w)=\frac{|w|_{v}}{|w|}$. In this section, we will show that

$$
\nu_{v}\left(\vartheta^{n}(a)\right)=\frac{\left|\vartheta^{n}(a)\right|_{v}}{\left|\vartheta^{n}(a)\right|} \stackrel{n \rightarrow \infty}{\longrightarrow} R_{v}^{(\ell)}
$$

holds almost surely, irrespective of the choice of $a \in \mathcal{A}$. To this end, we will split the word $\vartheta^{n}(a)=\vartheta^{k}\left(\vartheta^{n-k}(a)\right)$ into a large number of inflation words with level smaller than $n$. One technical obstacle is given by the fact that the (random) word $\vartheta^{n-k}(a)$ might comprise more than one type of letter, such that the action of $\vartheta^{k}$ on these letters cannot be interpreted as i.i.d. random words. This can be circumvented with the help of an elementary result. It basically establishes that if $\nu_{v}\left(\vartheta^{n}(u)\right)$ deviates from $R_{v}^{(\ell)}$ by a certain amount, then there is a letter $a \in \mathcal{A}$, comprising a positive fraction of $u$, such that a similar statement holds if we regard only the inflation words $\vartheta^{n}\left(u_{i}\right)$ for those letters $u_{i} \triangleleft u$ that coincide with $a$. We set up a slightly more general form for this result to allow for its application also in a different context.

Lemma 4.1. Let $c=\sum_{i=1}^{m} c_{i}$ and $d=\sum_{i=1}^{m} d_{i}$ with $0 \leqslant c_{i} \leqslant d_{i}$ and $1 \leqslant d_{i}$ for all $i \in\{1, \ldots, m\}$. Further, suppose that

$$
\left|\frac{c}{d}-K\right|>\delta
$$

for some $0 \leqslant \delta, K \leqslant 1$. Then, there exists some $j \in\{1, \ldots, m\}$ such that $d_{j} \geqslant \frac{\delta}{2 m} d$ and

$$
\left|\frac{c_{j}}{d_{j}}-K\right| \geqslant \frac{\delta}{2}
$$

Further, for this choice of $j$ and any $f \in \mathbb{R}_{+}$,

$$
\left|\frac{d_{j}}{f}-1\right| \geqslant \frac{\delta}{8} \quad \text { or } \quad\left|\frac{c_{j}}{f}-K\right| \geqslant \frac{\delta}{8} .
$$

Proof. Suppose Eq. (7) does not hold and set

$$
S_{1}=\left\{1 \leqslant i \leqslant m:\left|c_{i}-K d_{i}\right| \geqslant \frac{\delta}{2} d_{i}\right\}
$$

and $S_{2}=\{1, \ldots, m\} \backslash S_{1}$. Then, $i \in S_{1}$ implies $d_{i}<\frac{\delta}{2 m} d$. Note that generally $\left|c_{i}-K d_{i}\right| \leqslant d_{i}$. Consequently,

$$
|c-K d| \leqslant \sum_{i \in S_{1}}\left|c_{i}-K d_{i}\right|+\sum_{i \in S_{2}}\left|c_{i}-K d_{i}\right|<\sum_{i \in S_{1}} d_{i}+\sum_{i \in S_{2}} \frac{\delta}{2} d_{i}<\sum_{i \in S_{1}} \frac{\delta}{2 m} d+\frac{\delta}{2} d \leqslant \delta d,
$$

in contradiction to the assumption. This proves Eq. (7). Assuming $\left|d_{j} / f-1\right| \leqslant \delta / 8$, we obtain,

$$
\frac{\delta}{4} \leqslant \frac{d_{j}}{f} \frac{\delta}{2} \leqslant\left|\frac{c_{j}}{f}-K \frac{d_{j}}{f}\right| \leqslant\left|\frac{c_{j}}{f}-K\right|+K\left|1-\frac{d_{j}}{f}\right| \leqslant\left|\frac{c_{j}}{f}-K\right|+\frac{\delta}{8},
$$

and thus Eq. (8) holds. 


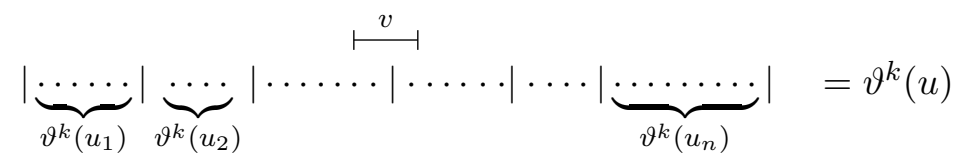

Figure 1. Example of a case where $v$ is not entirely included in any of the inflation words $\vartheta^{k}\left(u_{j}\right)$ but still occurs as a subword of $\vartheta^{k}(u)$.

Lemma 4.2. Le $v \in \mathcal{L}_{\vartheta}^{\ell}$ and $\varepsilon>0$. There is a $k_{0} \in \mathbb{N}$ such that, for all $k \geqslant k_{0}$, there is a $C>0$ and $n_{0} \in \mathbb{N}$ with the following property. If $n \geqslant n_{0}$, we have

$$
\mathbb{P}\left[\left|\nu_{v}\left(\vartheta^{k}(u)\right)-R_{v}^{(\ell)}\right|>\varepsilon\right] \leqslant \mathrm{e}^{-C n}
$$

for all $u \in \mathcal{L}_{\vartheta}^{n}$.

Proof. Let us start with some arbitrary $n \in \mathbb{N}$ and $u \in \mathcal{L}_{\vartheta}^{n}$. For any letter $a \in \mathcal{A}$, denote the positions of the letter $a$ within the word $u$ by $P_{a}=\left\{1 \leqslant i \leqslant n: u_{i}=a\right\}$, possibly empty, and $n_{a}=\operatorname{card} P_{a}=|u|_{a}$. Assume card $\mathcal{A}=m$ and

$$
\left|\frac{\left|\vartheta^{k}(u)\right|_{v}}{\left|\vartheta^{k}(u)\right|}-R_{v}^{(\ell)}\right|>\varepsilon
$$

Note that, in contrast to the total length $\left|\vartheta^{k}(u)\right|=\sum_{j=1}^{n}\left|\vartheta^{k}\left(u_{j}\right)\right|$, for the occurrences of $v$ within $\vartheta^{k}(u)$ it is not enough to count how often $v$ appears in any inflation word,

$$
\left|\vartheta^{k}(u)\right|_{v}=\sum_{j=1}^{n}\left|\vartheta^{k}\left(u_{j}\right)\right|_{v}+\mathrm{Ovl}
$$

where the additional term Ovl (in general a random variable) gives the number of times that the position of $v$ within $\vartheta^{k}(u)$ overlaps two (or more) neighbouring inflation words. A situation where such an overlap occurs is depicted in Figure 1. Since there are only $n-1$ different boundaries of inflation words of the form $\vartheta^{k}\left(u_{i}\right)$, the bound $0 \leqslant$ Ovl $<n \ell$ follows immediately. Setting $d=\left|\vartheta^{k}(u)\right|$ and $c=\sum_{j=1}^{n}\left|\vartheta^{k}\left(u_{j}\right)\right|_{v}$, one of the following two cases needs to hold,

$$
\text { (I) }\left|\frac{c}{d}-R_{v}^{(\ell)}\right|>\frac{\varepsilon}{2} \quad \text { or } \quad \text { (II) } \quad \ell n>\frac{\varepsilon}{2}\left|\vartheta^{k}(u)\right| .
$$

Let us first treat case (I). We want to translate this to a similar statement that involves only one type of letter as a starting point for the random inflation $\vartheta^{k}$; compare Figure 2 for an illustrative example. With the identifications $\delta=\frac{\varepsilon}{2}, K=R_{v}^{(\ell)}, c_{a}=\sum_{j \in P_{a}}\left|\vartheta^{k}\left(u_{j}\right)\right|_{v}$, $d_{a}=\sum_{j \in P_{a}}\left|\vartheta^{k}\left(u_{j}\right)\right|$ we can apply Lemma 4.1. That is, for all realisations of the random variables that we consider, there is some letter $a \in \mathcal{A}$ such that $\sum_{j \in P_{a}}\left|\vartheta^{k}\left(u_{j}\right)\right| \geqslant \frac{\varepsilon}{4 m}\left|\vartheta^{k}(u)\right|$ and (choosing $f=\lambda^{k} L_{a} n_{a}$, in the notation of Lemma 4.1),

$$
\left|\frac{1}{n_{a}} \sum_{j \in P_{a}} \frac{\left|\vartheta^{k}\left(u_{j}\right)\right|_{v}}{\lambda^{k} L_{a}}-R_{v}^{(\ell)}\right| \geqslant \frac{\varepsilon}{16} \quad \text { or } \quad\left|\frac{1}{n_{a}} \sum_{j \in P_{a}} \frac{\left|\vartheta^{k}\left(u_{j}\right)\right|}{\lambda^{k} L_{a}}-1\right| \geqslant \frac{\varepsilon}{16},
$$




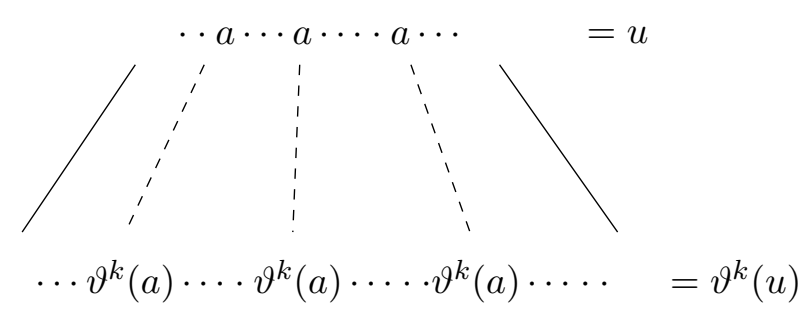

Figure 2. Inflation procedure of $\vartheta^{k}$ on $u$. The occurrences of some $a \in \mathcal{A}$ in $u$ are highlighted. These are mapped to i.i.d random words, distributed like $\vartheta^{k}(a)$, which appear as subwords of $\vartheta^{k}(u)$.

which we call case (Ia) and (Ib), respectively. Setting $\mu_{a}=\max \{k \in \mathbb{N}: \mathbb{P}[|\vartheta(a)|=k]>0\}$ and $\mu=\max _{a \in \mathcal{A}} \mu_{a}$ as the maximal possible inflation factor, we have the crude estimate,

$$
\mu^{k} n_{a} \geqslant \sum_{j \in P_{a}}\left|\vartheta^{k}\left(u_{j}\right)\right| \geqslant \frac{\varepsilon}{4 m}\left|\vartheta^{k}(u)\right| \geqslant \frac{\varepsilon}{4 m} n,
$$

that is, there is some constant $C_{1}(k, \varepsilon)=C_{1}>0$ such that $n_{a} \geqslant C_{1} n$. Let us define the random variables

$$
X_{(j)}^{k}=\frac{\left|\vartheta^{k}\left(u_{j}\right)\right|_{v}}{\lambda^{k} L_{a}} \text { and } Y_{(j)}^{k}=\frac{\left|\vartheta^{k}\left(u_{j}\right)\right|}{\lambda^{k} L_{a}}
$$

and note that each of the corresponding families with $j \in P_{a}$ comprise i.i.d. random variables with the same distribution $X^{k}$ and $Y^{k}$, respectively. By Lemma 3.5 and Proposition 3.8,

$$
\mathbb{E} Y_{(j)}^{k} \stackrel{k \rightarrow \infty}{\longrightarrow} 1 \text { and } \mathbb{E} X_{(j)}^{k} \stackrel{k \rightarrow \infty}{\longrightarrow} R_{v}^{(\ell)},
$$

for any $j \in P_{a}$. We can thus find a $k_{0}^{(1)} \in \mathbb{N}$ such that, for all $k \geqslant k_{0}^{(1)}$,

$$
\left|\frac{1}{n_{a}} \sum_{j \in P_{a}} X_{(j)}^{k}-\mathbb{E} X^{k}\right| \geqslant \frac{\varepsilon}{32} \quad \text { or } \quad\left|\frac{1}{n_{a}} \sum_{j \in P_{a}} Y_{(j)}^{k}-\mathbb{E} Y^{k}\right| \geqslant \frac{\varepsilon}{32} \text {. }
$$

Let us fix such a $k$. Then, the joint distribution of the family $\left(X_{(j)}^{k}\right)_{j \in P_{a}}$ does not depend on the structure of $u$ or the exact form of $P_{a}$, but only on $n_{a}=\operatorname{card} P_{a}$. Also, since $X^{k}$ can take only finitely many values, the bound $\mathbb{E} \mathrm{e}^{t X^{k}}<\infty$ is trivial for all $t \in \mathbb{R}$. By Cramér's theorem on large deviations [11, Thm. I.4], we therefore obtain,

$$
\lim _{n_{a} \rightarrow \infty} \frac{1}{n_{a}} \log \mathbb{P}\left[\left|\frac{1}{n_{a}} \sum_{j \in P_{a}} X_{(j)}^{k}-\mathbb{E} X^{k}\right| \geqslant \frac{\varepsilon}{32}\right]<0,
$$

and analogously for the family $\left(Y_{(j)}^{k}\right)_{j \in P_{a}}$. Thus, for large enough $n$ and any $u \in \mathcal{L}_{n}$,

$$
\mathbb{P}[(\mathrm{I})] \leqslant \sum_{a \in \mathcal{A}} \mathbb{P}[(\mathrm{Ia}) \text { holds for } a]+\sum_{a \in \mathcal{A}} \mathbb{P}[(\mathrm{Ib}) \text { holds for } a] \leqslant \sum_{a \in \mathcal{A}} \mathrm{e}^{-\widetilde{C} n_{a}} \leqslant|\mathcal{A}| \mathrm{e}^{-\widetilde{C} C_{1} n} .
$$

We now turn to case (II). This can be rephrased as

$$
\frac{\sum_{a \in \mathcal{A}}|u|_{a}}{\sum_{a \in \mathcal{A}} \sum_{j \in P_{a}}\left|\vartheta^{k}\left(u_{j}\right)\right|}>\frac{\varepsilon}{2 \ell},
$$


such that we can again apply Lemma 4.1, this time with $\delta=\frac{\varepsilon}{2 \ell}, K=0, c_{a}=\left|u_{a}\right|$ and $d_{a}=\sum_{j \in P_{a}}\left|\vartheta^{k}\left(u_{j}\right)\right|$, yielding the existence of some $a \in \mathcal{A}$ with $\sum_{j \in P_{a}}\left|\vartheta^{k}\left(u_{j}\right)\right| \geqslant \frac{\epsilon}{4 \ell m}\left|\vartheta^{k}(u)\right|$ and, recalling $n_{a}=|u|_{a}$,

$$
\frac{1}{n_{a}} \sum_{j \in P_{a}}\left|\vartheta^{k}\left(u_{j}\right)\right|<\frac{4 \ell}{\varepsilon} .
$$

Note that all the random words $\vartheta^{k}\left(u_{j}\right)$ are distributed as $\vartheta^{k}(a)$ and again i.i.d. for $j \in P_{a}$. At the same time,

$$
n_{a}>\frac{\varepsilon}{4 \ell} \sum_{j \in P_{a}}\left|\vartheta^{k}\left(u_{j}\right)\right| \geqslant \frac{\varepsilon^{2}}{16 \ell^{2} m}\left|\vartheta^{k}(u)\right| \geqslant \frac{\varepsilon^{2}}{16 \ell^{2} m} n .
$$

Since $\mathbb{E}\left|\vartheta^{k}\left(u_{j}\right)\right| \stackrel{k \rightarrow \infty}{\longrightarrow} \infty$, as $k \rightarrow \infty$, we can find a $k_{0}^{(2)}$ such that, for all $k \geqslant k_{0}^{(2)}$, Eq. (9) implies

$$
\frac{1}{n_{a}} \sum_{j \in P_{a}}\left|\vartheta^{k}\left(u_{j}\right)\right|<\frac{1}{2} \mathbb{E}\left|\vartheta^{k}(a)\right| .
$$

Similarly to case (I), we can apply basic large deviation results to conclude that the probability for this to happen is bounded by $\mathrm{e}^{-C_{2} n}$, for some $C_{2}>0$ and $n \in \mathbb{N}$ large enough.

Finally, taking $k_{0}=\max \left\{k_{0}^{(1)}, k_{0}^{(2)}\right\}$ and adjusting the constant in the exponent, $k \geqslant k_{0}$ implies

$$
\mathbb{P}\left[\left|\nu_{v}\left(\vartheta^{k}(u)\right)-R_{v}^{(\ell)}\right|>\varepsilon\right] \leqslant \mathbb{P}[(\mathrm{I})]+\mathbb{P}[(\mathrm{II})] \leqslant \mathrm{e}^{-C n} .
$$

for large enough $n \in \mathbb{N}$.

Proposition 4.3. Let $v \in \mathcal{L}_{\vartheta}^{\ell}$ be a $\vartheta$-legal word of length $\ell \in \mathbb{N}$. Then, in the limit of large inflation words, the word frequency of $v$ exists and is the same for almost all realisations. It coincides with the corresponding entry $R_{v}^{(\ell)}$ of the right PF eigenvector of the induced substitution matrix. More precisely, for any $a \in \mathcal{A}$,

$$
\lim _{n \rightarrow \infty} \nu_{v}\left(\vartheta^{n}(a)\right)=R_{v}^{(\ell)}
$$

holds almost surely.

Proof. Let $\varepsilon>0$ and $a \in \mathcal{A}$. We are going to establish that

$$
\sum_{n=1}^{\infty} \mathbb{P}\left[\left|\nu_{v}\left(\vartheta^{n}(a)\right)-R_{v}^{(\ell)}\right|>\varepsilon\right]<\infty .
$$

From this, the assertion follows by a standard application of the Borel-Cantelli lemma.

Due to Proposition 3.6, there are some $\widetilde{C}, K>0$ such that $\mathbb{P}\left[\left|\vartheta^{n}(a)\right|<K n\right] \leqslant \mathrm{e}^{-\widetilde{C} n}$ for large enough $n \in \mathbb{N}$. Choose $C>0$ and a fixed $k \geqslant k_{0}$ as in Lemma 4.2. Then, for large enough $n \in \mathbb{N}$,

$$
\begin{aligned}
\mathbb{P}\left[\left|\nu_{v}\left(\vartheta^{k+n}(a)\right)-R_{v}^{(\ell)}\right|>\varepsilon\right] & \leqslant \mathrm{e}^{-\tilde{C} n}+\sum_{u \in \mathcal{L},|u| \geqslant K n} \mathbb{P}\left[\left|\nu_{v}\left(\vartheta^{k}(u)\right)-R_{v}^{(\ell)}\right|>\varepsilon\right] \mathbb{P}\left[\vartheta^{n}(a)=u\right] \\
& \leqslant \mathrm{e}^{-\widetilde{C} n}+\mathrm{e}^{-C K n},
\end{aligned}
$$

by Lemma 4.2. This verifies Eq. (10). 
Remark 4.4. The statement of Proposition 4.3 for the special case $\ell=1$ (frequency of letters) follows from a standard result on branching processes. More precisely, performing the Abelianisation, we obtain a vector-valued Markov process $\left(\Phi\left(\vartheta^{n}(a)\right)\right)_{n \in \mathbb{N}}$ which forms a multitype Galton-Watson (GW) process as described in [18] and [1]. From [1, Ch. V.6], we find that, for every $a \in \mathcal{A}$,

$$
\lim _{n \rightarrow \infty} \frac{\Phi\left(\vartheta^{n}(a)\right)}{\lambda^{n}}=R W_{a}
$$

holds almost surely, where $W_{a}$ is a non-negative random variable with $\mathbb{E}\left[W_{a}\right]=L_{a}$ and $\mathbb{P}\left[W_{a}=0\right]=0$. Note that $\left(\Phi_{\ell}\left(\vartheta_{\ell}^{n}(a)\right)\right)_{n \in \mathbb{N}}$ for $\ell \geqslant 2$ does not form a multitype GW process, because neighbouring words have some overlap which is necessarily mapped to the same word under $\vartheta$. This violates the requirement for a $\mathrm{GW}$ process that each individual produces offspring independently. However, combining Proposition 4.3 with Eq. (11) we obtain that for any $a \in \mathcal{A}$ and $v \in \mathcal{L}_{\vartheta}^{\ell}$,

$$
\lim _{n \rightarrow \infty} \frac{\left|\vartheta^{n}(a)\right|_{v}}{\lambda^{n}}=\lim _{n \rightarrow \infty} \frac{\left|\vartheta^{n}(a)\right|_{v}}{\left|\vartheta^{n}(a)\right|} \frac{\left|\vartheta^{n}(a)\right|}{\lambda^{n}}=R_{v}^{(\ell)} W_{a}
$$

holds almost surely. Note that, despite our preceding words of precaution, this result (and thereby Proposition 4.3) can also be obtained by generalising ideas from the theory of GW processes [23, Thm. 2].

\section{ERgodic Frequency MEASURES ON THE HULL}

From the word frequencies in inflation words, as established in the last section, we want to define a shift-invariant measure on the hull $\mathbb{X}_{\vartheta}$ of the substitution in a consistent way. We obtain this by defining the measure first on a convenient class of subsets. A cylinder set on $\mathbb{X}_{\vartheta}$ is of the form $\mathcal{Z}_{[k, m]}(v)=\left\{x \in \mathbb{X}_{\vartheta}: x_{[k, m]}=v\right\}$, for some $k \leqslant m \in \mathbb{Z}$ and $v \in \mathcal{L}_{\vartheta}^{m-k+1}$. We call $\mathcal{B}_{\vartheta}$ the Borel sigma-algebra with respect to the topology generated by the cylinder sets.

Remark 5.1. It is worth noticing that $\mathcal{B}_{\vartheta}$ is already generated by the smaller class of those cylinder sets that specify the position 0 :

$$
\mathfrak{Z}_{0}\left(\mathbb{X}_{\vartheta}\right)=\left\{\mathcal{Z}_{[k, m]}(v): k \leqslant 0 \leqslant m, v \in \mathcal{L}_{\vartheta}^{m-k+1}\right\} \cup\left\{\mathbb{X}_{\vartheta}, \varnothing\right\} .
$$

The class $\mathfrak{Z}_{0}\left(\mathbb{X}_{\vartheta}\right)$ has the convenient property of forming a semi-algebra on $\mathbb{X}$ (it contains the full space, is stable under intersections, and complements can be written as finite disjoint unions).

Definition 5.2. A map $\mu: \mathfrak{S} \rightarrow[0, \infty]$ on a semi-algebra $\mathfrak{S}$ is called a measure on $\mathfrak{S}$, if the following conditions hold:

(1) If $\bigcup_{i=1}^{\infty} A_{i} \in \mathfrak{S}$ is a disjoint union of elements $A_{i} \in \mathfrak{S}$, then $\mu\left(\bigcup_{i=1}^{\infty} A_{i}\right)=\sum_{i=1}^{\infty} \mu\left(A_{i}\right)$,

(2) $\mu(\varnothing)=0$.

Often, this is also called a premeasure in the literature. We know from [22, Cor. 2.4.9] and [22, Prop. 2.5.1] that this can be extended uniquely to a measure on the $\sigma$-algebra generated by $\mathfrak{S}$ if $\mu$ is $\sigma$-finite on $\mathfrak{S}$. Comparing this with Remark 5.1, we find that it is enough to specify a measure on $\mathfrak{Z}_{0}\left(\mathbb{X}_{\vartheta}\right)$ in order to uniquely define a measure on $\left(\mathbb{X}_{\vartheta}, \mathcal{B}_{\vartheta}\right)$. 
Proposition 5.3. Let $\mu_{\vartheta}: \mathfrak{Z}_{0}\left(\mathbb{X}_{\vartheta}\right) \rightarrow[0,1]$ be defined via $\mu_{\vartheta}(\varnothing)=0, \mu_{\vartheta}\left(\mathbb{X}_{\vartheta}\right)=1$ and $\mu_{\vartheta}\left(\mathcal{Z}_{[k, m]}(v)\right)=R_{v}^{(\ell)}$, for any $k \leqslant 0 \leqslant m \in \mathbb{Z}$ and $v \in \mathcal{L}_{\vartheta}^{\ell}$ with $|v|=m-k+1$. Here, $R^{(\ell)}$ denotes the right PF-eigenvector of the induced substitution $\vartheta_{\ell}$. Then, $\mu_{\vartheta}$ defines a $\sigma$-finite measure on $\mathfrak{Z}_{0}\left(\mathbb{X}_{\vartheta}\right)$ and thereby also on $\left(\mathbb{X}_{\vartheta}, \mathcal{B}_{\vartheta}\right)$.

As a first step, we establish a consistency relation for the word frequencies which ensures the finite additivity of $\mu_{\vartheta}$.

Lemma 5.4. Suppose $v=v_{1} \cdots v_{\ell_{0}} \in \mathcal{L}_{\vartheta}$, and fix an arbitrary $\ell \in \mathbb{N}$, with $\ell \geqslant \ell_{0}$ and a position $k \in\left\{1, \ldots, \ell-\ell_{0}+1\right\}$. If $\left.\mathcal{G}_{k}^{\ell}(v)=\left\{u \in \mathcal{L}_{\vartheta}^{\ell}: u_{\left[k, k+\ell_{0}-1\right.}\right]=v\right\}$, then

$$
R_{v}^{\left(\ell_{0}\right)}=\sum_{u \in \mathcal{G}_{k}^{\ell}(v)} R_{u}^{(\ell)}
$$

Proof. Fix $\omega \in \Omega$ and $a \in \mathcal{A}$ such that $\nu_{u}\left(\vartheta^{n}(a)(\omega)\right)$ converges to $R_{u}^{(|u|)}$ as $n \rightarrow \infty$, for all $u \in \mathcal{L}_{\vartheta}$. Now,

$$
\left|\vartheta^{n}(a)(\omega)\right|_{v}=\operatorname{card}\left\{j \in\left\{1, \ldots,\left|\vartheta^{n}(a)(\omega)\right|-\ell_{0}+1\right\}: \vartheta^{n}(a)(\omega)_{\left[j, j+\ell_{0}-1\right]}=v\right\} .
$$

Note that, for $j \geqslant k, \vartheta^{n}(a)(\omega)_{\left[j, j+\ell_{0}-1\right]}=v$ if and only if $\vartheta^{n}(a)(\omega)_{[j-k+1, j-k+\ell]}=u$ for some $u \in \mathcal{G}_{k}^{\ell}(v)$, as long as $j-k+\ell \leqslant\left|\vartheta^{n}(a)(\omega)\right|$. That is, if we count the cardinality of the corresponding set, we miss at most $\ell-\ell_{0}$ subwords of type $v$ in $\vartheta^{n}(a)(\omega)$. More precisely, with $i=j-k$,

$$
\begin{aligned}
\left|\vartheta^{n}(a)(\omega)\right|_{v} & =\operatorname{card} \bigcup_{u \in \mathcal{G}_{k}^{\ell}(v)}\left\{i \in\left\{0, \ldots,\left|\vartheta^{n}(a)(\omega)\right|-\ell\right\}: \vartheta^{n}(a)(\omega)_{[i+1, i+\ell]}=u\right\}+\mathcal{O}\left(\ell-\ell_{0}\right) \\
& =\sum_{u \in \mathcal{G}_{k}^{\ell}(v)}\left|\vartheta^{n}(a)(\omega)\right|_{u}+\mathcal{O}\left(\ell-\ell_{0}\right) .
\end{aligned}
$$

Dividing both sides by $\left|\vartheta^{n}(a)(\omega)\right|$ and taking the limit $n \rightarrow \infty$ yield the desired relation.

Proof of Proposition 5.3. First, note that every $\mathcal{Z} \in \mathfrak{Z}_{0}\left(\mathbb{X}_{\vartheta}\right)$ is compact as a closed subset of a compact space. Hence, every countable disjoint union $\dot{\bigcup}_{i=1}^{\infty} \mathcal{Z}_{i}=\mathcal{Z} \in \mathfrak{Z}_{0}\left(\mathbb{X}_{\vartheta}\right)$ is in fact finite, that is $\dot{\bigcup}_{i=1}^{\infty} \mathcal{Z}_{i}=\dot{\bigcup}_{j \in I} \mathcal{Z}_{j}$ for some finite index set $I$. It thus suffices to show that $\mu_{\vartheta}$ is finitely additive on $\mathfrak{Z}_{0}\left(\mathbb{X}_{\vartheta}\right)$.

We can rewrite any cylinder set $\mathcal{Z}_{[j, m]}(v)$ as a union of cylinder sets that specify a larger set of positions

$$
\mathcal{Z}_{[j, m]}(v)=\bigcup_{u \in \mathcal{G}_{k}^{2 n+1}(v)}^{\cdot} \mathcal{Z}_{[-n, n]}(u),
$$

for any $n \geqslant \max \{|j|,|m|\}$ and $k=j+n+1$. Lemma 5.4 yields

$$
\mu_{\vartheta}\left(\mathcal{Z}_{[j, m]}(v)\right)=R_{v}^{(m-j+1)}=\sum_{u \in \mathcal{G}_{k}^{2 n+1}(v)} R_{u}^{(2 n+1)}=\sum_{u \in \mathcal{G}_{k}^{2 n+1}} \mu_{\vartheta}\left(\mathcal{Z}_{[-n, n]}(u)\right),
$$

providing the additivity of $\mu_{\vartheta}$ on disjoint unions of the form in Eq. (12). The same kind of relation holds for $\mathcal{Z}=\mathbb{X}_{\vartheta}$ if we replace $\mathcal{G}_{k}^{2 n+1}(v)$ by $\mathcal{L}_{\vartheta}^{2 n+1}$. 
The finite additivity of $\mu_{\vartheta}$ on disjoint unions of cylinder sets of the form $\dot{\bigcup}_{j \in I} \mathcal{Z}_{j}=\mathcal{Z}$ follows in a straightforward manner. Just consider a common refinement into cylinder sets of the form $\mathcal{Z}_{[-n, n]}(u)$ for large enough $n \in \mathbb{N}$. Hence, $\mu_{\vartheta}$ is a $(\sigma-)$ finite measure on $\mathfrak{Z}_{0}\left(\mathbb{X}_{\vartheta}\right)$.

Remark 5.5. It is clear by definition that $\mu_{\vartheta}$ is shift-invariant on the cylinder sets of the semi-algebra $\mathfrak{Z}_{0}\left(\mathbb{X}_{\vartheta}\right)$. This also implies that $\mu_{\vartheta}$ is shift-invariant as a measure on $\left(\mathbb{X}_{\vartheta}, \mathcal{B}_{\vartheta}\right)$ compare [31, Thm. 1.1]. We are therefore left with a shift invariant measure space $\left(\mathbb{X}_{\vartheta}, \mathcal{B}_{\vartheta}, \mu_{\vartheta}\right)$.

As an important step towards ergodicity, we give a sufficient condition for the almost sure convergence of word frequencies with respect to the measure $\mu_{\vartheta}$. For the following, we fix an arbitrary averaging sequence of intervals $I_{n}=\left[a_{n}, b_{n}\right]$ with $a_{n} \leqslant b_{n}$ and $a_{n}, b_{n} \in \mathbb{Z}$ for all $n \in \mathbb{N}$ such that the length of the intervals $d_{n}=b_{n}-a_{n}+1$ is strictly monotonously increasing.

Lemma 5.6. Let $\left(\mathbb{X}_{\vartheta}, \mathcal{B}_{\vartheta}, \mu_{\vartheta}\right)$ be the measure space introduced above. For a given word $v \in \mathcal{L}_{\vartheta}^{\ell}$ and $\varepsilon>0$, let $C_{\varepsilon}^{n}:=\left\{u \in \mathcal{L}_{\vartheta}^{n}:\left|\nu_{v}(u)-R_{v}^{(\ell)}\right|>\varepsilon\right\}$. Suppose that, for every $\varepsilon>0$,

$$
\sum_{n=1}^{\infty} \sum_{u \in C_{\varepsilon}^{n}} R_{u}^{(n)}<\infty
$$

Then, for almost every element $y \in \mathbb{X}_{\vartheta}$, the frequency of $v$ in $y$ with respect to the averaging sequence $\left\{\left[a_{n}, b_{n}\right]\right\}_{n \in \mathbb{N}}$ is well-defined and given by $R_{v}^{(\ell)}$. That is, there exists a set of full measure $A^{v} \subseteq \mathbb{X}_{\vartheta}$ such that, for any $y \in A^{v}$, we have

$$
\lim _{n \rightarrow \infty} \nu_{v}\left(y_{\left[a_{n}, b_{n}\right]}\right)=R_{v}^{(\ell)} \text {. }
$$

Proof. Consider the random variables

$$
X_{n}: \mathbb{X}_{\vartheta} \rightarrow \mathbb{R}, \quad y \mapsto \nu_{v}\left(y_{\left[a_{n}, b_{n}\right]}\right)=\frac{\left|y_{\left[a_{n}, b_{n}\right]}\right|_{v}}{d_{n}},
$$

for all $n \in \mathbb{N}$, and the constant random variable $X: \mathbb{X}_{\vartheta} \rightarrow \mathbb{R}, y \mapsto R_{v}^{(\ell)}$. It is a well-known fact (by an application of the Borel-Cantelli lemma) that $\left(X_{n}\right)_{n \in \mathbb{N}}$ converges $\mu_{\vartheta}$-almost surely to $X$ if

$$
\sum_{n=1}^{\infty} \mu_{\vartheta}\left(\left\{y \in \mathbb{X}_{\vartheta}|| X_{n}(y)-X(y) \mid>\varepsilon\right\}\right)<\infty,
$$

for every $\varepsilon>0$. Therefore, our claim follows from

$$
\begin{aligned}
\mu_{\vartheta}\left(\left\{y \in \mathbb{X}_{\vartheta}|| X_{n}(y)-X(y) \mid>\varepsilon\right\}\right) & =\mu_{\vartheta}\left(\left\{y \in \mathbb{X}_{\vartheta} \mid y_{\left[a_{n}, b_{n}\right]} \in C_{\varepsilon}^{d_{n}}\right\}\right) \\
& =\mu_{\vartheta}\left(\left\{y \in \mathbb{X}_{\vartheta} \mid y \in \bigcup_{u \in C_{\varepsilon}^{d_{n}}} \mathcal{Z}_{\left[a_{n}, b_{n}\right]}(u)\right\}\right) \\
& =\mu_{\vartheta}\left(\bigcup_{u \in C_{\varepsilon}^{d_{n}}} \mathcal{Z}_{\left[a_{n}, b_{n}\right]}(u)\right)=\sum_{u \in C_{\varepsilon}^{d_{n}}} \mu_{\vartheta}\left(\mathcal{Z}_{\left[a_{n}, b_{n}\right]}(u)\right) \\
& =\sum_{u \in C_{\varepsilon}^{d_{n}}} R_{u}^{\left(d_{n}\right)} \leqslant \sum_{u \in C_{\varepsilon}^{n}} R_{u}^{(n)},
\end{aligned}
$$

where the last step is a consequence of the assumption that $\left(d_{n}\right)_{n \in \mathbb{N}}$ is a strictly increasing sequence in $\mathbb{N}$. 
Remark 5.7. The proof of the ergodicity result for a family of random noble means subsitutions in [20] follows a somewhat different approach. There, for a given $\ell \in \mathbb{N}_{0}$ and $k, m \in \mathbb{Z}$, the subwords $X_{[k, k+\ell]}$ and $X_{[m, m+\ell]}$ of a $\mu_{\vartheta}$-distributed random word $X$ are interpreted as i.i.d. random words, provided that $k$ and $m$ are separated by a certain distance. The small gap in the proof, mentioned in the introduction, amounts to the fact that independence for the given, fixed distance does not hold and should be replaced by asymptotic independence as the distance approaches infinity. However, the varied correlation structure of different examples of random substitutions precludes using a similar approach in the general case. We therefore speak of i.i.d. random words only in the context of the Markov measure $\mathbb{P}$ and use the estimate in Lemma 4.2 to relate this to properties of $\mu_{\vartheta}$.

Next, we want to show that Eq. (14) indeed holds for any expansive primitive random substitution. The intuition behind this is the following. For a word $u$ to be in $C_{\varepsilon}^{n}$, it must be exceptional regarding the occurrences of the subword $v$. The sum of the frequencies of such words $u$ can not be too large since otherwise it would contradict the well-defined frequency of $v$ in the typical limit words of the random substitution Markov process. The idea is to exhaust exceptional words $u$ with inflation words and to show that $u \in C_{\varepsilon}^{n}$ essentially requires a positive fraction of these inflation words to have exceptional relative words frequencies as well. We can then employ the large deviation estimates in Lemma 4.2 to show that these events must have summable probabilities.

Proposition 5.8. Let $v \in \mathcal{L}_{\vartheta}^{\ell}$ be a $\vartheta$-legal word of length $\ell$ and $\left\{I_{n}\right\}_{n \in \mathbb{N}}$ an averaging sequence of strictly increasing length. Then, for $\mu_{\vartheta}$-almost every element $y \in \mathbb{X}_{\vartheta}$, the frequency of $v$ in $y$ with respect to $\left\{I_{n}\right\}_{n \in \mathbb{N}}$ is well-defined and given by $R_{v}^{(\ell)}$.

Proof. It suffices to show that Eq. (14) indeed holds for all $\varepsilon>0$. Let $v \in \mathcal{L}_{\vartheta}^{\ell}$ be fixed in the following. Because of Proposition 3.3, it is for $x, u \in \mathcal{L}_{\vartheta}^{n}$ and $k \in \mathbb{N}:\left(M_{\vartheta_{n}}^{k}\right)_{x u}=\mathbb{E} \Phi_{n}\left(\vartheta_{n}^{k}(u)\right)_{x}$. Hence, we can rewrite the frequency of $x \in C_{\varepsilon}^{n}$ as

$R_{x}^{(n)}=\frac{1}{\lambda^{k}}\left(M_{\vartheta_{n}}^{k} R^{(n)}\right)_{x}=\frac{1}{\lambda^{k}} \sum_{u \in \mathcal{L}_{\vartheta}^{n}} \mathbb{E} \Phi_{n}\left(\vartheta_{n}^{k}(u)\right)_{x} R_{u}^{(n)}=\sum_{u \in \mathcal{L}_{\vartheta}^{n}} R_{u}^{(n)} \underbrace{\frac{1}{\lambda^{k}} \sum_{V \in \mathcal{D}_{n}} \mathbb{P}\left[\vartheta_{n}^{k}(u)=V\right]|V|_{x}}_{=: Q_{n}^{k}(u, x)}$,

for any $k \in \mathbb{N}$. Thus, with $Q_{n}^{k}\left(u, C_{\varepsilon}^{n}\right):=\sum_{x \in C_{\varepsilon}^{n}} Q_{n}^{k}(u, x)$, it is

$$
\sum_{x \in C_{\varepsilon}^{n}} R_{x}^{(n)}=\sum_{u \in \mathcal{L}_{\vartheta}^{n}} R_{u}^{(n)} Q_{n}^{k}\left(u, C_{\varepsilon}^{n}\right) .
$$

Given a large enough $k$, our strategy is to find a bound for $Q_{n}^{k}\left(u, C_{\varepsilon}^{n}\right)$, uniform in $u$, that is small enough to be summable over $n$. Note that

$$
\begin{aligned}
Q_{n}^{k}\left(u, C_{\varepsilon}^{n}\right) & =\frac{1}{\lambda^{k}} \sum_{V \in \mathcal{D}_{n}} \sum_{j=1}^{|V|} \mathbb{P}\left[\vartheta_{n}^{k}(u)=V\right] \delta_{V_{j}}\left(C_{\varepsilon}^{n}\right) \\
& =\frac{1}{\lambda^{k}} \sum_{m=1}^{\infty} \sum_{j=1}^{m} \mathbb{P}\left[\vartheta_{n}^{k}(u)_{j} \in C_{\varepsilon}^{n} \wedge\left|\vartheta_{n}^{k}(u)\right|=m\right] .
\end{aligned}
$$




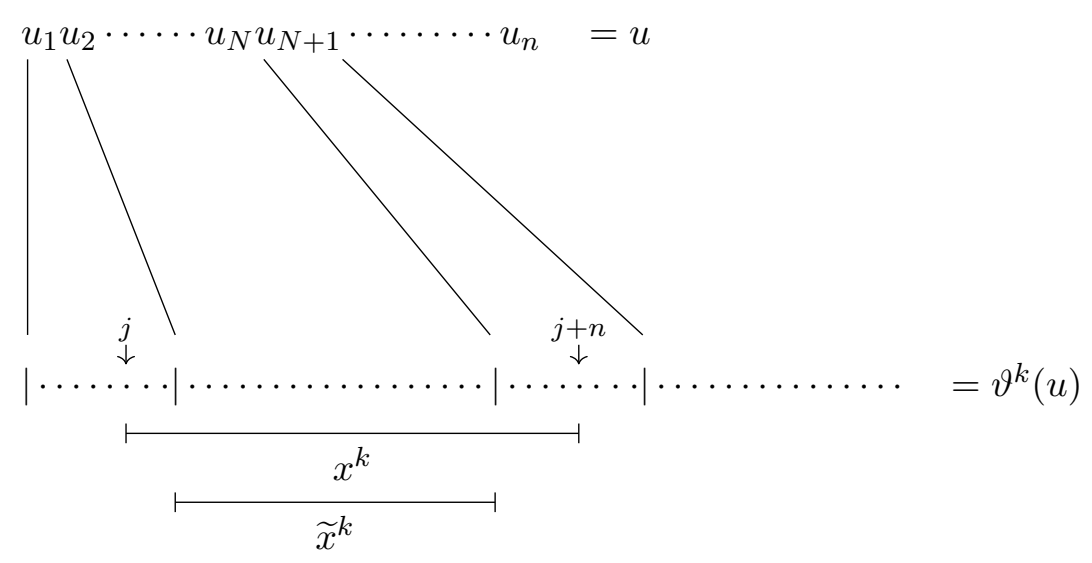

Figure 3. Illustration of how the random words $x^{k}, N=N_{k}$ and $\widetilde{x}^{k}$ are built from $\vartheta^{k}(u)$, given a fixed length $n \in \mathbb{N}$, a number of inflation steps $k \in \mathbb{N}$ and an initial position $1 \leqslant j \leqslant\left|\vartheta^{k}\left(u_{1}\right)\right|$.

This is still a finite sum, since $\left|\vartheta_{n}^{k}(u)\right|$ can take only finitely many values. We can exchange the order of summation and express this as

$$
Q_{n}^{k}\left(u, C_{\varepsilon}^{n}\right)=\frac{1}{\lambda^{k}} \sum_{j=1}^{\infty} \mathbb{P}\left[\vartheta_{n}^{k}(u)_{j} \in C_{\varepsilon}^{n} \wedge\left|\vartheta_{n}^{k}(u)\right| \geqslant j\right] .
$$

Now, by definition, $\left|\vartheta_{n}^{k}(u)(\omega)\right|=\left|\vartheta^{k}\left(u_{1}\right)(\omega)\right|$ and $\vartheta_{n}^{k}(u)(\omega)_{j}=\vartheta^{k}(u)(\omega)_{[j, j+n-1]}$ for every realisation $\omega$. Thus,

$$
Q_{n}^{k}\left(u, C_{\varepsilon}^{n}\right)=\frac{1}{\lambda^{k}} \sum_{j=1}^{\mu^{k}} \mathbb{P}\left[\vartheta^{k}(u)_{[j, j+n-1]} \in C_{\varepsilon}^{n} \wedge\left|\vartheta^{k}\left(u_{1}\right)\right| \geqslant j\right],
$$

where $\mu=\max _{\omega \in \Omega} \max _{a \in \mathcal{A}}|\vartheta(a)(\omega)|$ is an upper bound for the 1-step inflation factor. Note that, at this point, $k \in \mathbb{N}$ is still a free parameter.

Let us go back to Eq. (17). For the bulk of the remainder, we fix a position $j \in \mathbb{N}_{0}$ and consider the random variable given by $x^{k}=\vartheta^{k}(u)_{[j, j+n-1]}$, under the additional condition that $\left|\vartheta^{k}\left(u_{1}\right)\right| \geqslant j$. This ensures that $x^{k}$ overlaps the inflation word $\vartheta^{k}\left(u_{0}\right)$. Note that, in general, $x^{k}$ depends only on a prefix of $u$, given by $u_{\left[1, N_{k}+1\right]}$, where we take $N_{k} \in \mathbb{N}_{0}$ to be minimal with this property. Clearly, $N_{k}$ depends on the chosen realisation of $x^{k}$. It is thus also a random variable and we may regard it as a stopping time. By the minimality of $N_{k}$, we obtain that

$$
\vartheta^{k}\left(u_{\left[2, N_{k}(\omega)\right]}\right)(\omega) \triangleleft x^{k}(\omega) \triangleleft \vartheta^{k}\left(u_{\left[1, N_{k}(\omega)+1\right]}\right)(\omega)
$$

holds for every realisation $\omega$. Note that the 'interior' of $x^{k}$ on the left hand side is a concatenation of inflation words

$$
\widetilde{x}^{k}:=\vartheta^{k}\left(u_{\left[2, N_{k}\right]}\right)=\vartheta^{k}\left(u_{2}\right) \cdot \ldots \cdot \vartheta^{k}\left(u_{N_{k}}\right),
$$

suppressing the dependence on $\omega$ in our notation. Compare Figure 3 for a graphical representation of those quantities. Note that the ratio of $\left|\widetilde{x}^{k}\right|$ and $n$ approaches 1 as $n \rightarrow \infty$ since 


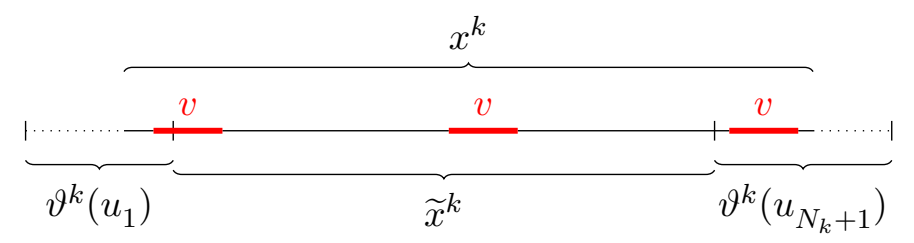

Figure 4. Possible positions of the word $v$ (highlighted in red) as a subword of $x^{k}$.

$\left|\vartheta^{k}(a)\right| \leqslant \mu^{k}$ for all $a \in \mathcal{A}$ (recall that $\mu$ denotes the maximal length of level-1 inflation words) and thus

$$
\frac{n-2 \mu^{k}}{n} \leqslant \frac{n-\left|\vartheta^{k}\left(u_{0}\right)\right|-\left|\vartheta^{k}\left(u_{N_{k}+1}\right)\right|}{n} \leqslant \frac{\left|\widetilde{x}^{k}\right|}{n} \leqslant 1 .
$$

Consider the event $x^{k} \in C_{\varepsilon}^{n}$ that is equivalent to

$$
\left|\frac{\left|x^{k}\right|_{v}}{n}-R_{v}^{(\ell)}\right|>\varepsilon
$$

We wish to express this in terms of the exact inflation word $\widetilde{x}^{k}$. We can split up the occurrences of $v$ in $x^{k}$ into those that are contained in $\widetilde{x}^{k}$, those contained in the overlap of $x^{k}$ with the boundary inflation words $\vartheta^{k}\left(u_{1}\right)$ or $\vartheta^{k}\left(u_{N_{t}+1}\right)$ and finally those $v$ that overlap both $\widetilde{x}^{k}$ and one of the boundary inflation words. For an illustration of the three qualitatively different positions of $v$ within $x^{k}$, compare Figure 4. Thus,

$$
\left|\widetilde{x}^{k}\right|_{v} \leqslant\left|x^{k}\right|_{v} \leqslant\left|\widetilde{x}^{k}\right|_{v}+\left|\vartheta^{k}\left(u_{1}\right)\right|_{v}+\left|\vartheta^{k}\left(u_{N_{k}+1}\right)\right|_{v}+2 \ell .
$$

The last term originates from the fact that there are two boundaries of $\widetilde{x}^{k}$ with boundary inflation words each of which can contribute at most $|v|=\ell$ occurrences of $v$ to the sum. For large enough $n$, we therefore conclude that

$$
\left|\frac{\left|x^{k}\right|_{v}}{n}-\frac{\left|\widetilde{x}^{k}\right|_{v}}{\left|\widetilde{x}^{k}\right|}\right| \leqslant\left|\frac{\left|x^{k}\right|_{v}}{n}-\frac{\left|\widetilde{x}^{k}\right|_{v}}{n}\right|+\left|\frac{\left|\widetilde{x}^{k}\right|_{v}}{n}-\frac{\left|\widetilde{x}^{k}\right|_{v}}{\left|\widetilde{x}^{k}\right|}\right| \leqslant \frac{\varepsilon}{2}
$$

such that Eq. (18) implies

$$
\left|\nu_{v}\left(\vartheta^{k}\left(u_{\left[2, N_{k}\right]}\right)\right)-R_{v}^{(\ell)}\right|=\left|\frac{\left|\widetilde{x}^{k}\right|_{v}}{\left|\widetilde{x}^{k}\right|}-R_{v}^{(\ell)}\right|>\frac{\varepsilon}{2} .
$$

Corresponding to $\frac{\varepsilon}{2}$, let us fix some $k \geqslant k_{0}$ as in Lemma 4.2. Note that $N_{k}$ grows linearly with $n$ because

$$
N_{k} \leqslant n \leqslant\left|\vartheta^{k}\left(u_{\left[1, N_{k}+1\right]}\right)\right| \leqslant \mu^{k}\left(N_{k}+1\right),
$$

that is, there is some $C_{1}>0$ such that $C_{1} n \leqslant N_{k}(\omega) \leqslant n$ for large enough $n$, uniformly for all realisations $\omega \in \Omega$. An application of Lemma 4.2 yields

$$
\begin{aligned}
\mathbb{P}[(18)] & \leqslant \mathbb{P}[(19)]=\sum_{N=C_{1} n}^{n} \mathbb{P}\left[\left|\nu_{v}\left(\vartheta^{k}\left(u_{[2, N]}\right)\right)-R_{v}^{(\ell)}\right|>\frac{\varepsilon}{2} \wedge N_{k}=N\right] \\
& \leqslant \sum_{N=C_{1} n}^{n} \mathrm{e}^{-C N} \leqslant n \mathrm{e}^{-C C_{1} n} \leqslant \mathrm{e}^{-\widetilde{C} n}
\end{aligned}
$$


for large enough $n$ and appropriate choice of $\widetilde{C}>0$. Since this bound holds irrespective of the choice of $j$, we have for any $j \in\left\{1, \ldots, \mu^{k}\right\}$,

$$
\mathbb{P}\left[\vartheta^{k}(u)_{[j, j+n-1]} \in C_{\varepsilon}^{n} \wedge\left|\vartheta^{k}\left(u_{1}\right)\right| \geqslant j\right] \leqslant \mathbb{P}[(18)] \leqslant \mathrm{e}^{-\widetilde{C} n} .
$$

Comparing with Eq. (17), we find

$$
Q_{n}^{k}\left(u, C_{\varepsilon}^{n}\right) \leqslant \frac{\mu^{k}}{\lambda^{k}} \mathrm{e}^{-\widetilde{C} n} \leqslant \mathrm{e}^{-C^{\prime} n}
$$

for $n$ larger than some $n_{0}$, by another adjustment of the constant. Since this is summable over $n$, we find, using Eq. (16),

$$
\sum_{n=1}^{\infty} \sum_{x \in C_{\varepsilon}^{n}} R_{x}^{(n)} \leqslant \sum_{n=1}^{n_{0}} \sum_{x \in C_{\varepsilon}^{n}} R_{x}^{(n)}+\sum_{n=n_{0}+1}^{\infty} \underbrace{\sum_{u \in \mathcal{L}_{\vartheta}^{n}} R_{u}^{(n)}}_{=1} \mathrm{e}^{-C^{\prime} n}<\infty .
$$

This finishes the proof.

Finally, we are ready to prove the main result of this paper.

Theorem 5.9. Let $\vartheta$ be an expansive, primitive random substitution. Then, the measuretheoretic dynamical system $\left(\mathbb{X}_{\vartheta}, \mathcal{B}_{\vartheta}, \mu_{\vartheta}\right)$ is ergodic.

Proof. It suffices to show that the identity

$$
\lim _{N \rightarrow \infty} \frac{1}{N} \sum_{j=0}^{N-1} f\left(S^{j} x\right)=\int_{\mathbb{X}_{\vartheta}} f \mathrm{~d} \mu_{\vartheta}
$$

holds for every $f \in L^{1}\left(\mathbb{X}_{\vartheta}, \mathcal{B}_{\vartheta}, \mu_{\vartheta}\right)$ and almost all $x \in \mathbb{X}_{\vartheta}$.

First, let $\mathcal{Z} \in \mathfrak{Z}_{0}\left(\mathbb{X}_{\vartheta}\right)$ be a cylinder set and $f=1_{\mathcal{Z}}$ be the indicator function on $\mathcal{Z}$. More

precisely, let $\mathcal{Z}=\mathcal{Z}_{[k, m]}(v)$, where $k \leqslant m \in \mathbb{Z}$, and $v \in \mathcal{L}_{\vartheta}^{m-k+1}$. Now, by definition of $\mu_{\vartheta}$ and Proposition 5.8, we obtain

$$
\int_{\mathbb{X}_{\vartheta}} f \mathrm{~d} \mu_{\vartheta}=\mu_{\vartheta}\left(\mathcal{Z}_{[k, m]}(v)\right)=R_{v}^{(m-k+1)}=\lim _{N \rightarrow \infty} \frac{\left|x_{[k, m+N-1]}\right| v}{N}=\lim _{N \rightarrow \infty} \frac{1}{N} \sum_{j=0}^{N-1} f\left(S^{j} x\right),
$$

for almost all $x$. Hence, Eq. (20) is true if $f$ is the indicator function on a cylinder set.

Second, let $\Gamma:=\left\{\sum_{\mathcal{Z} \in S} a_{\mathcal{Z}} 1_{\mathcal{Z}} \mid S \subseteq \mathfrak{Z}_{0}\left(\mathbb{X}_{\vartheta}\right)\right.$ is finite and $\left.a_{\mathcal{Z}} \in \mathbb{C}\right\}$ be the set of simple functions on $\left(\mathbb{X}_{\vartheta}, \mathcal{B}_{\vartheta}, \mu_{\vartheta}\right)$. By linearity, the validity of Eq. (20) for indicator functions on cylinder sets extends to arbitrary functions in $\Gamma$.

Third, since $\Gamma$ is dense in $L^{1}\left(\mathbb{X}_{\vartheta}, \mathcal{B}_{\vartheta}, \mu_{\vartheta}\right)$, the claim follows.

\section{UNIQUE, STRICT AND INTRINSIC ERGODICITY}

The last part of this paper is devoted to studying further properties of the ergodic measures $\mu_{\vartheta}$ that we constructed in the preceding section. It turns out that random substitutions are a general enough class of objects to allow for a variety of different behaviours. We illustrate this by a number of examples. First, let us show that, in general, the measures $\mu_{\vartheta}$ are not uniquely ergodic. 
Example 6.1. Consider the random period doubling substitution

$$
\sigma: a \mapsto\left\{\begin{array}{ll}
a b . & \text { with probability } p \\
b a, & \text { with probability } q:=1-p
\end{array}, \quad b \mapsto a a,\right.
$$

for some $0<p<1$. A short computation gives

$$
M_{\sigma}=\left(\begin{array}{ll}
1 & 2 \\
1 & 0
\end{array}\right) \quad \text { and } \quad M_{\sigma_{2}}=\left(\begin{array}{cccc}
p q & q & 1+p & 2 \\
1-p q & p & q & 0 \\
1-p q & 1 & 0 & 0 \\
p q & 0 & 0 & 0
\end{array}\right) \text {. }
$$

Hence, we obtain $R^{(2)}=\left(\frac{2}{3\left(p^{2}-p+2\right)}, \frac{2\left(1-p+p^{2}\right)}{3\left(p^{2}-p+2\right)}, \frac{2\left(1-p+p^{2}\right)}{3\left(p^{2}-p+2\right)}, \frac{p-p^{2}}{3\left(p^{2}-p+2\right)}\right)^{\top}$. Therefore,

$$
\int_{\mathbb{X}_{\sigma}} 1_{\mathcal{Z}_{0}(b b)} \mathrm{d} \mu_{\sigma}=\mu_{\sigma}\left(\mathcal{Z}_{0}(b b)\right)=R_{b b}^{(2)}=\frac{p-p^{2}}{3\left(p^{2}-p+2\right)}>0 .
$$

On the other hand, we have

$$
\frac{1}{N} \sum_{k=0}^{N-1} 1_{\mathcal{Z}_{0}(b b)}\left(S^{k} x\right)=0
$$

for all $N \in \mathbb{N}$ if $x$ is an element of the hull of the deterministic period doubling substitution (which is a subset of the random hull). Consequently, Eq. (20) does not hold for all $x \in \mathbb{X}_{\sigma}$ and thus $\mu_{\sigma}$ is not uniquely ergodic.

In fact, it is possible to characterise those random substitution subshifts which are uniquely ergodic - compare [28, Thm. 27(b) and Cor. 28] for a proof of the following result.

Proposition 6.2. Let $\left(\mathbb{X}_{\vartheta}, S\right)$ be a primitive random substitution subshift.

(a) $\left(\mathbb{X}_{\vartheta}, S\right)$ is uniquely ergodic if and only if the right PF eigenvectors $R^{(\ell)}$ are independent of the probability vectors $\boldsymbol{p}_{i}$, for every $\ell$ and every $i$.

(b) If $\left(\mathbb{X}_{\vartheta}, S\right)$ is uniquely ergodic, it is also strictly ergodic.

Most interesting examples of random substitutions however, give rise to highly non-minimal subshifts $\mathbb{X}$; see [28]. In that case, we can find an uncountable family of ergodic frequency measures with full support on $\mathbb{X}$. Let us expand a bit on this point.

Recall that for a random substitution rule $P^{\vartheta}$, the subshift $\mathbb{X}_{\vartheta}$ depends only on the family $\left(\operatorname{supp} P_{a}^{\vartheta}\right)_{a \in \mathcal{A}}$. For each $a \in \mathcal{A}$, the values of $P_{a}^{\vartheta}$ on its support can therefore be regarded as free parameters with the additional constraint of forming a probability vector $\boldsymbol{p}_{a}$ on $\operatorname{supp} P_{a}^{\vartheta}$ with non-zero entries. Given any $\ell \in \mathbb{N}$, the entries of $M_{\vartheta_{\ell}}$ are polynomials in the entries of $\boldsymbol{p}_{a}$ for $a \in \mathcal{A}$ by construction and thus $R^{(\ell)}$ depends continuously on those parameters, compare $R^{(2)}$ in Example 6.1. Assuming $R^{(\ell)}$ is not constant in all of the $\boldsymbol{p}_{a}$, it thus takes a continuum of values giving rise to a continuum of different ergodic frequency measures on $\mathbb{X}_{\vartheta}$. We summarise this observation as follows.

Corollary 6.3. Let $\mathbb{X}$ be a random substitution subshift. For any pair $\vartheta, \vartheta^{\prime}$ of expanding primitive random substitution generating $\mathbb{X}=\mathbb{X}_{\vartheta}=\mathbb{X}_{\vartheta^{\prime}}$, the frequency measures $\mu_{\vartheta}$ and $\mu_{\vartheta^{\prime}}$ are either equal or mutually singular. If $(\mathbb{X}, S)$ is not uniquely ergodic, there is an uncountable set of random substitutions $\Theta$ such that $\mu_{\vartheta} \perp \mu_{\vartheta^{\prime}}$ for all $\vartheta, \vartheta^{\prime} \in \Theta$ with $\vartheta \neq \vartheta^{\prime}$. 
Next, let us focus on the question whether or not a primitive random substitution subshift can be intrinsically ergodic, that is whether it allows a unique measure which maximises the entropy. We know that there is at least one measure of maximal entropy, see [6, Sec. (17.15), Cor. 2]. Consider the following two examples.

Example 6.4. Let $\mathbb{X}$ be a topologically transitive shift of finite type. It was shown in [10] that $\mathbb{X}$ can be realised as a primitive RS-subshift. Since every topologically transitive shift of finite type is intrinsically ergodic [21], there are intrinsically ergodic RS-subshifts. At present, it remains open whether the measure of maximal entropy on $\mathbb{X}$ can be realised as a frequency measure $\mu_{\vartheta}$ arising from some random substitution $\vartheta$.

Example 6.5. The Dyck shift is a coded shift, which is not sofic. Moreover, it has two measures of maximal entropy [14]. Hence, it is not intrinsically ergodic. The Dyck shift can be realised as a primitive RS-subshift via the random substitution

$$
(\mapsto\{((),([],(\}, \quad[\mapsto\{[(),[[],[\}, \quad) \mapsto\{()),[]),)\}, \quad] \mapsto\{[]],()],]\},
$$

where the assignment of (non-degnerate) probability vectors is arbitrary. Therefore, not every RS-subshift is intrinsically ergodic.

To get a better feeling, let us compute the metric entropy for a specific example. To do so, we need the following result, which follows by an application of standard techniques from linear algebra; compare [30, Thm. 1.1, Cor. 1].

Lemma 6.6. Let $M \in \operatorname{Mat}(d, \mathbb{R})$ be a primitive matrix such that $M_{i j}=m_{i} \in \mathbb{R}_{\geq 0}$ for all $i, j \in\{1, \ldots, d\}$. Then, $\lambda=\sum_{i=1}^{d} m_{i}$ is the PF eigenvalue of $M$, and the corresponding (normalised) right $P F$ eigenvector is given by

$$
R=\frac{1}{\lambda}\left(m_{1}, \ldots, m_{d}\right)^{\top}
$$

Proposition 6.7. Consider the random substitution

$$
\zeta: a \mapsto\left\{\begin{array}{ll}
a b, & \text { with probability } p \\
b a, & \text { with probability } 1-p
\end{array}, \quad b \mapsto\left\{\begin{array}{ll}
a b, & \text { with probability } p \\
b a, & \text { with probability } 1-p
\end{array},\right.\right.
$$

for $0<p<1$. If $\mu$ denotes the corresponding frequency measure, the metric entropy $h_{\mu}$ is given by

$$
h_{\mu}=-\frac{1}{2}(p \log (p)+(1-p) \log (1-p)) .
$$

Proof. By definition of the metric entropy and the frequency measures, we obtain from Lemma 6.6

$$
\begin{aligned}
h_{\mu} & =\lim _{n \rightarrow \infty}-\frac{1}{n} \sum_{w \in \mathcal{L}_{\zeta}^{n}} \mu([w]) \cdot \log (\mu([w]))=\lim _{n \rightarrow \infty}-\frac{1}{n} \sum_{w \in \mathcal{L}_{\zeta}^{n}} R_{w}^{(n)} \cdot \log \left(R_{w}^{(n)}\right) \\
& =\lim _{n \rightarrow \infty}-\frac{1}{n} \sum_{i=1}^{\left|\mathcal{L}_{\zeta}^{n}\right|} \frac{1}{\lambda} m_{i}^{(n)} \cdot \log \left(\frac{1}{\lambda} m_{i}^{(n)}\right)=\frac{1}{\lambda} \cdot \lim _{n \rightarrow \infty}-\frac{1}{n} \sum_{i=1}^{\left|\mathcal{L}_{\zeta}^{n}\right|} m_{i}^{(n)} \cdot \log \left(m_{i}^{(n)}\right),
\end{aligned}
$$


where $m_{i}^{(n)}=\mathbb{E}\left|\zeta_{n}(u)\right|_{u_{i}}$, for $u, u_{i} \in \mathcal{L}_{\zeta}^{n}$, is independent of $u$. From now on, we consider the subsequence $\left(m_{i}^{(2 n)}\right)_{n \in \mathbb{N}}$. Let $u=u_{1} \ldots u_{2 n} \in \mathcal{L}_{\zeta}^{2 n}$. Then, for all $\omega \in \Omega, \zeta(u)(\omega)$ is of the form

$$
\zeta(u)(\omega)=\underset{k=1}{\stackrel{2 n}{\odot}} v^{k}, \quad v^{k} \in\{a b, b a\}
$$

where $\odot$ denotes concatenation of words from left to right. Therefore,

$$
\zeta_{2 n}(u)(\omega)=\left(v^{1} \ldots v^{n}\right)\left(v_{2}^{1} v^{2} \ldots v^{n} v_{1}^{n+1}\right) .
$$

This implies

$$
\mathbb{E}\left[\left|\zeta_{2 n}(u)\right|_{u_{i}}\right]=\sum_{k=1}^{2} \mathbb{E}\left[\delta_{u_{i}}\left(\zeta(u)_{[k, k+2 n-1]}\right)\right]=\sum_{k=1}^{2} \mathbb{P}\left[\zeta(u)_{[k, k+2 n-1]}=u_{i}\right] .
$$

Note that, for a specific choice of the $v^{k}, k \in\{1, \ldots, n\}$,

$$
\mathbb{P}\left[\zeta(u)_{[1,2 n]}=\stackrel{\bigodot_{k=1}^{n}}{v^{k}}\right]=p^{\left|\left\{k \mid v^{k}=a b\right\}\right|} \cdot(1-p)^{\left|\left\{k \mid v^{k}=b a\right\}\right|} .
$$

Since the suffix and prefix $v_{2}^{1}$ and $v_{1}^{n+1}$ determine the inflation words $v^{1}$ and $v^{n+1}$ uniquely, we also have that

$$
\mathbb{P}\left[\zeta(u)_{[2,2 n+1]}=v_{2}^{1}\left(\underset{k=2}{\odot} v^{k}\right) v_{1}^{n+1}\right]=p^{\left|\left\{k \mid v^{k}=a b\right\}\right|} \cdot(1-p)^{\left|\left\{k \mid v^{k}=b a\right\}\right|},
$$

where $k$ ranges from 1 to $n+1$ on the right hand side. For $u_{i}$ to be legal, it must either be of the form $u_{i}=v^{1} \cdots v^{n}$ or $u_{i}=v_{2}^{1} v^{2} \cdots v^{n} v_{1}^{n+1}$, determining the words $v^{k} \in\{a b, b a\}$ uniquely in either case. The only words that can be written in both forms are $u_{a}=a b a b \ldots a b$ and $u_{b}=b a b a \ldots b a$. Note that there are exactly $\left(\begin{array}{l}n \\ j\end{array}\right)$ different words of the form $u=v^{1} \cdots v^{n}$ with $\left|\left\{k=1, \ldots, n \mid v^{k}=a b\right\}\right|=j$ for every $j \in\{0, \ldots, n\}$ and $\left(\begin{array}{c}n+1 \\ j\end{array}\right)$ pairwise different words of the form $u_{i}=v_{2}^{1} v^{2} \cdots v^{n} v_{1}^{n+1}$ with $\left|\left\{k=0, \ldots, n \mid v^{k}=a b\right\}\right|=j$ for all $j \in\{0, \ldots, n+1\}$. Together with Eq. (22), we obtain

$$
\begin{aligned}
\sum_{i=1}^{\left|\mathcal{L}_{\zeta}^{2 n}\right|} m_{i}^{(2 n)} \cdot \log \left(m_{i}^{(2 n)}\right)= & \sum_{j=0}^{n}\left(\begin{array}{c}
n \\
j
\end{array}\right) p^{j}(1-p)^{n-j} \log \left(p^{j}(1-p)^{n-j}\right) \\
& +\sum_{j=0}^{n+1}\left(\begin{array}{c}
n+1 \\
j
\end{array}\right) p^{j}(1-p)^{n+1-j} \log \left(p^{j}(1-p)^{n+1-j}\right) \\
& \quad+\operatorname{Err}_{2 n} \\
= & \log (p)(2 n+1) p+\log (1-p)(2 n+1)(1-p)+\operatorname{Err}_{2 n}
\end{aligned}
$$

where the error term $\operatorname{Err}_{2 n}=\operatorname{Err}_{2 n}^{a}+\operatorname{Err}_{2 n}^{b}$, with

$$
\begin{aligned}
& \operatorname{Err}_{2 n}^{a}=\left(p^{n}+(1-p)^{n+1}\right) \log \left(p^{n}+(1-p)^{n+1}\right)-p^{n} \log \left(p^{n}\right)-(1-p)^{n+1} \log \left((1-p)^{n+1}\right) \\
& \operatorname{Err}_{2 n}^{b}=\left(p^{n+1}+(1-p)^{n}\right) \log \left(p^{n+1}+(1-p)^{n}\right)-p^{n+1} \log \left(p^{n+1}\right)-(1-p)^{n} \log \left((1-p)^{n}\right)
\end{aligned}
$$




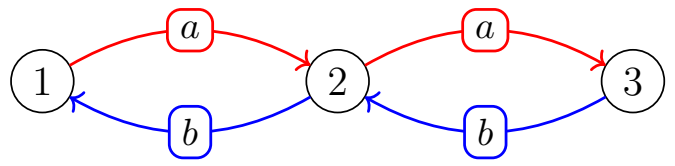

Figure 5. The right-resolving graph representation of $X_{\zeta}$ as a sofic shift.

accounts for the fact that there are two words $u_{a}$ and $u_{b}$ for which both summands in Eq. (22) are non-zero. Clearly, we have $\frac{1}{2 n} \operatorname{Err}_{2 n} \rightarrow 0$ for $n \rightarrow \infty$. Hence, we conclude

$$
\begin{aligned}
h_{\mu} & =-\frac{1}{\lambda} \cdot \lim _{n \rightarrow \infty} \frac{1}{2 n}\left(\log (p)(2 n+1) p+\log (1-p)(2 n+1)(1-p)+\operatorname{Err}_{2 n}\right) \\
& =-\frac{1}{2}(p \log (p)+(1-p) \log (1-p)),
\end{aligned}
$$

since $\lambda=2$.

Corollary 6.8. Let $\zeta$ be as in the previous proposition. Then, the $R S$-subshift $\mathbb{X}_{\zeta}$ is intrinsically ergodic, and the frequency measure which corresponds to $p=\frac{1}{2}$ is the measure of maximal entropy.

Proof. It is not difficult to see that the subshift generated by the graph in Figure 5 coincides with $\mathbb{X}_{\zeta}$. Therefore, it is a sofic shift and thus intrinsically ergodic.

The topological entropy of $\mathbb{X}_{\zeta}$ is given by $h\left(\mathbb{X}_{\zeta}\right)=\frac{1}{2} \log (2)$ which follows from simple combinatorics on $\mathbb{X}_{\zeta}$; alternatively, see [8] for a criterion that allows to read off $h\left(\mathbb{X}_{\zeta}\right)$ immediately from the form of the random substitution. Now, if $p=\frac{1}{2}$, the previous proposition implies that $h_{\mu}=\frac{1}{2} \log (2)$, which finishes the proof.

Consequently, if $\boldsymbol{p}=(p, 1-p)^{\top}$ is the uniform distribution, we obtain the measure of maximal entropy. This result also holds for a larger class of random substitutions. For this, consider the random substitution

$$
\vartheta: a_{i} \mapsto\left\{\begin{array}{cc}
w^{1}, & \text { with probability } p_{1} \\
\vdots & \vdots \\
w^{\ell}, & \text { with probability } p_{\ell}
\end{array} \quad \text { for all } a_{i} \in \mathcal{A},\right.
$$

where $\ell \in \mathbb{N}, \boldsymbol{p}=\left(p_{1}, \ldots, p_{\ell}\right)^{\top}$ is a probability vector, and $w^{1}, \ldots, w^{\ell}$ are legal words of length $N \in \mathbb{N}$, such that $\vartheta$ is primitive and $\left|w^{j_{1}}\right|_{a_{i}}=\left|w^{j_{2}}\right|_{a_{i}}$ for all $j_{1}, j_{2} \in\{1, \ldots, \ell\}$ and for all $a_{i} \in \mathcal{A}$.

Proposition 6.9. Let $\vartheta$ be as above. Then, $\mathbb{X}_{\vartheta}$ is intrinsically ergodic. Moreover, the frequency measure corresponding to the vector $\boldsymbol{p}=\left(\frac{1}{\ell}, \ldots, \frac{1}{\ell}\right)^{\top}$ is the measure of maximal entropy.

Proof. The subshift $\mathbb{X}_{\vartheta}$ is a sofic shift, and therefore it is intrinsically ergodic; compare the previous proposition. 
By Eq. (21), the metric entropy can be computed via

$$
h_{\mu}=\lim _{n \rightarrow \infty}-\frac{1}{n} \sum_{i=1}^{\left|\mathcal{L}_{\vartheta}^{n}\right|} \frac{1}{\lambda} m_{i}^{(n)} \cdot \log \left(\frac{1}{\lambda} m_{i}^{(n)}\right)=\lim _{n \rightarrow \infty}-\frac{1}{n} \sum_{i=1}^{\left|\mathcal{L}_{\vartheta}^{n}\right|} \frac{1}{N} m_{i}^{(n)} \cdot \log \left(\frac{1}{N} m_{i}^{(n)}\right),
$$

since $\lambda=N$ is the PF eigenvalue of $M_{\vartheta}$. Let us consider the subsequence $(N n)_{n \in \mathbb{N}}$ of $(n)_{n \in \mathbb{N}}$. Let $u=u_{1} \ldots u_{N n} \in \mathcal{L}_{\vartheta}^{N n}$. Then, we have for all $\omega \in \Omega$

$$
\vartheta(u)(\omega)=\underset{j=1}{N n} v^{j}, \quad v^{j} \in\left\{w^{1}, \ldots, w^{\ell}\right\}
$$

by the inflation-word structure and thus

$$
\vartheta_{N n}(u)(\omega)=\left(v^{1} \ldots v^{n}\right)\left(v_{[2, N]}^{1} v^{2} \ldots v^{n} v_{1}^{n+1}\right) \ldots\left(v_{N}^{1} v^{2} \ldots v^{n} v_{[1, N-1]}^{n+1}\right) .
$$

due to the fact that all $v^{j}$ have the same length $N$. Analogously to Eq. (22), we obtain

$$
m_{i}^{(N n)}=\sum_{k=1}^{N} \mathbb{P}\left[\vartheta(u)_{[k, k+N n-1]}=u_{i}\right]
$$

Since every inflation word $w^{j}, j=1, \ldots, \ell$ appears with the same probability $\frac{1}{\ell}$, we find that, for all $1 \leqslant k \leqslant N$ and $u_{i} \in \mathcal{L}_{\vartheta}^{n}$, the term $\mathbb{P}\left[\vartheta(u)_{[k, k+N n-1]}=u_{i}\right]$ is either 0 or

$$
\ell^{-2} \ell^{-(n-1)} \leqslant \mathbb{P}\left[\vartheta(u)_{[k, k+N n-1]}=u_{i}\right] \leqslant \ell^{-(n-1)} .
$$

Also, since $u_{i}$ is legal, at least one of the terms needs to be non-zero. This implies

$$
\frac{1}{N} \ell^{-2} \ell^{-(n-1)} \leqslant \frac{1}{N} m_{i}^{(N n)} \leqslant \ell^{-(n-1)}
$$

and

$$
1=\sum_{i=1}^{\left|\mathcal{L}_{\vartheta}^{N n}\right|} \frac{1}{N} m_{i}^{(N n)} \in\left[\left|\mathcal{L}_{\vartheta}^{N n}\right| \frac{1}{N} \ell^{-2} \ell^{-(n-1)},\left|\mathcal{L}_{\vartheta}^{N n}\right| \ell^{-(n-1)}\right]
$$

which leads to

$$
\frac{1}{N} \ell^{-2} \ell^{-(n-1)} \leqslant \frac{1}{\left|\mathcal{L}_{\vartheta}^{N n}\right|} \leqslant \ell^{-(n-1)}
$$

This together with Eq. (23) gives

$$
c_{1} \frac{1}{\left|\mathcal{L}_{\vartheta}^{N n}\right|} \leqslant \frac{1}{N} m_{i}^{(N n)} \leqslant c_{2} \frac{1}{\left|\mathcal{L}_{\vartheta}^{N n}\right|},
$$

where $c_{1}:=\frac{1}{N \ell^{2}}$ and $c_{2}:=N \ell^{2}$. That is, the entries of the right eigenvector $R^{(N n)}$ can differ from the uniform distribution only by a non-zero factor, which can be chosen independent of $n$. This is enough to conclude that the metric entropy coincides with the topological entropy. 
More precisely, we have

$$
\begin{aligned}
h_{\mu} & =\lim _{n \rightarrow \infty}-\frac{1}{N n} \sum_{i=1}^{\left|\mathcal{L}_{\vartheta}^{N n}\right|} \frac{1}{N} m_{i}^{(N n)} \log \left(\frac{1}{N} m_{i}^{(N n)}\right) \\
& \geqslant \lim _{n \rightarrow \infty}-\frac{1}{N n} \sum_{i=1}^{\left|\mathcal{L}_{\vartheta}^{N n}\right|} \frac{1}{N} m_{i}^{(N n)} \log \left(c_{1} \frac{1}{\left|\mathcal{L}_{\vartheta}^{N n}\right|}\right) \\
& =\lim _{n \rightarrow \infty}-\frac{1}{N n} \log \left(\frac{1}{\left|\mathcal{L}_{\vartheta}^{N n}\right|}\right)=h\left(\mathbb{X}_{\vartheta}\right),
\end{aligned}
$$

i.e. $h_{\mu} \geqslant h\left(\mathbb{X}_{\vartheta}\right)$. Since one always has $h_{\mu} \leqslant h\left(\mathbb{X}_{\vartheta}\right)$, the claim follows.

Similarly as in Corollary 6.8, this allows us to conclude

Corollary 6.10. Let $\vartheta$ be as above, and denote by $\mu$ the measure of maximal entropy, i.e. the frequency measure that corresponds to the vector $\boldsymbol{p}=\left(\frac{1}{\ell}, \ldots, \frac{1}{\ell}\right)^{\top}$. Then,

$$
h_{\mu}=\frac{1}{N} \log (\ell)
$$

Remark 6.11. Proposition 6.9 gives an alternative characterisation of the measure of maximal entropy for a certain class of RS-subshifts, which are sofic shifts. Numerical computations suggest that this result can be extended to include non-sofic subshifts, such as the RS-subshift which arises from the random Fibonacci substitution

$$
\vartheta_{\text {Fib }}: a \mapsto\left\{\begin{array}{ll}
b a, & \text { with probability } p \\
a b, & \text { with probability } 1-p
\end{array}, \quad b \mapsto a .\right.
$$

The difficulty in this situation is the computation of the normalised right PF eigenvectors $R^{(n)}$ for large $n$.

\section{Appendix}

In this section we will make explicit a possible choice of the probability spaces involved in the construction of a random substitution $\vartheta$ and the corresponding Markov chain. Let us define $\mathcal{I}_{0}=\{\langle a p\rangle: a \in \mathcal{A}, p \in \mathbb{N}\}$. We call an element $I=\langle a p\rangle \in \mathcal{I}_{0}$ an individual of type $a$ and position $p$. To each individual $I$ we attach a copy of $\mathcal{A}^{+}$and define the product space $\Omega_{2}=X_{I \in \mathcal{I}_{0}}\left(\mathcal{A}^{+}\right)_{I}$. We equip $\mathcal{A}^{+}$with the discrete topology, $\Omega$ with the corresponding product topology and define $\mathcal{F}_{2}$ to be the sigma-algebra of Borel sets. If $I=\langle a p\rangle$, we define $P_{I}=P_{a}^{\vartheta}$ and from this, we construct the product measure $\mathbb{P}_{2}=\bigotimes_{I \in \mathcal{I}_{0}} P_{I}$. This yields a probability space $\left(\Omega_{2}, \mathcal{F}_{2}, \mathbb{P}_{2}\right)$. Now, suppose $\mathcal{U}$ is a random word on $\left(\Omega_{1}, \mathcal{F}_{1}, \mathbb{P}_{1}\right)$ and define $(\Omega, \mathcal{F}, \mathbb{P})=\left(\Omega_{1} \times \Omega_{2}, \mathcal{F}_{1} \otimes \mathcal{F}_{2}, \mathbb{P}_{1} \otimes \mathbb{P}_{2}\right)$. For a given $\omega=\left(\omega_{1}, \omega_{2}\right) \in \Omega$, let $u=u_{1} \cdots u_{m}=$ $\mathcal{U}(\omega)=\mathcal{U}\left(\omega_{1}\right)$. From this, we define a subset of selected individuals $\mathcal{I}_{0}^{\omega}=\left\{\left\langle u_{1} 1\right\rangle, \ldots,\left\langle u_{m} m\right\rangle\right\}$. For each $j \in\{1, \ldots, m\}, \omega_{\left\langle u_{j} j\right\rangle}$ is a component of $\omega_{2}=\left(\omega_{I}\right)_{I \in \mathcal{I}_{0}}$. Finally,

$$
\vartheta(\mathcal{U})(\omega):=\omega_{\left\langle u_{1} 1\right\rangle} \cdots \omega_{\left\langle u_{m} m\right\rangle} \in \mathcal{A}^{+} .
$$


Lemma 7.1. The random words $\mathcal{U}$ and $\vartheta(\mathcal{U})$ on $(\Omega, \mathcal{F}, \mathbb{P})$ as defined above satisfy

$$
\mathbb{P}[\vartheta(\mathcal{U})=v \mid \mathcal{U}=u]=P(u, v),
$$

for all $u, v \in \mathcal{A}^{+}$.

Proof. Assume $\omega \in \Omega$ such that $\mathcal{U}(\omega)=u=u_{1} \cdots u_{m}$. Then, $\vartheta(\mathcal{U})(\omega)=\omega_{\left\langle u_{1} 1\right\rangle} \cdots \omega_{\left\langle u_{m} m\right\rangle}$, by definition. Since $\mathbb{P}$ is a product measure, we obtain

$$
\begin{aligned}
\mathbb{P}[\vartheta(\mathcal{U})=v \mid \mathcal{U}=u] & =\mathbb{P}\left[\omega_{\left\langle u_{1} 1\right\rangle} \cdots \omega_{\left\langle u_{m} m\right\rangle}=v\right]=\sum_{v^{1} \cdots v^{m}=v} \mathbb{P}\left[\omega_{\left\langle u_{1} 1\right\rangle}=v^{1}\right] \cdots \mathbb{P}\left[\omega_{\left\langle u_{m} m\right\rangle}=v^{m}\right] \\
& =\sum_{v^{1} \cdots v^{m}=v} P_{u_{1}}^{\vartheta}\left(v^{1}\right) \cdots P_{u_{m}}^{\vartheta}\left(v^{m}\right)=P(u, v),
\end{aligned}
$$

where we have used $\sum_{v^{1} \ldots v^{m}=v}$ as a shorthand notation for $\sum_{v^{1}, \ldots, v^{m} \in \mathcal{A}^{+}}$.

In order to iterate $\vartheta$ we might in principle always take the product with the same probability space $\left(\Omega_{2}, \mathcal{F}_{2}, \mathbb{P}_{2}\right)$, outlined above. However, it will prove convenient to equip the individuals in subsequent 'generations' with a bit more structure. The following construction is along the same lines as for the multitype Galton-Watson process, compare [18, Ch. 1.2]. We focus on the case that we start from a deterministic word $u=u_{1} \cdots u_{m} \in \mathcal{A}^{+}$. We form a set of initial individuals $\mathcal{I}_{0}=\left\{\left\langle u_{1} 1\right\rangle, \ldots,\left\langle u_{m} m\right\rangle\right\}$. An individual of generation $n$ is a tuple $I=\left\langle a_{0} p_{0} a_{1} p_{1} \ldots a_{n} p_{n}\right\rangle$ with $n \in \mathbb{N},\left\langle a_{0} p_{0}\right\rangle \in \mathcal{I}_{0}, a_{i} \in \mathcal{A}$ and $p_{i} \in \mathbb{N}$ for all $1 \leqslant i \leqslant n$. We call $a(I):=a_{n}$ the type of the individual and $p_{n}$ its position. We say that $I=\left\langle a_{0} p_{0} \cdots a_{k} p_{k}\right\rangle$ is an ancestor of $J=\left\langle a_{0}^{\prime} p_{0}^{\prime} \cdots a_{k+s}^{\prime} p_{k+s}^{\prime}\right\rangle$ if $a_{j}=a_{j}^{\prime}$ and $p_{j}=p_{j}^{\prime}$ for all $1 \leqslant j \leqslant k$. For example, we want to think of $\left\langle a_{0} p_{0} a_{1} p_{1}\right\rangle$ as an individual of type $a_{1}$ produced from $\left\langle a_{0} p_{0}\right\rangle$ and occurring at position $p_{1}$ in the word that $\left\langle a_{0} p_{0}\right\rangle$ is substituted with. The collection of all possible individuals forms a countable set

$$
\mathcal{I}=\bigcup_{n \geqslant 0} \mathcal{I}_{n}, \quad \mathcal{I}_{n}=\left\{\left\langle a_{0} p_{0} \cdots a_{n} p_{n}\right\rangle:\left\langle a_{0} p_{0}\right\rangle \in \mathcal{I}_{0}, a_{i} \in \mathcal{A}, p_{i} \in \mathbb{N} \text { for all } 1 \leqslant i \leqslant n\right\} .
$$

From this, we define $\Omega_{u}=\times_{I \in \mathcal{I}}\left(\mathcal{A}^{+}\right)_{I}$ with Borel sigma-algebra $\mathcal{B}_{u}$ and probability measure $\mathbb{P}_{u}=\bigotimes_{I \in \mathcal{I}} P_{I}$. Again, $P_{I}=P_{a}^{\vartheta}$, where $a$ is the type of $I$. On finite cylinders of the form $B=\prod_{I} B_{I}$ where $B_{I} \neq \mathcal{A}^{+}$only for $I$ in a finite subset $\mathcal{J} \subset \mathcal{I}$, this measure is given by

$$
\mathbb{P}\left(\prod_{I} B_{I}\right)=\prod_{I \in \mathcal{J}} P_{I}\left(B_{I}\right) .
$$

In the process that we want to model, only a finite subset of $\mathcal{I}_{n}$ is produced from the previous generation in each step. For $\omega=\left(\omega_{I}\right)_{I \in \mathcal{I}} \in \Omega_{u}$, the set of selected individuals $\mathcal{I}_{n}^{\omega} \subset \mathcal{I}_{n}$ for each generation $n$ is defined inductively. First, $\mathcal{I}_{0}^{\omega} \equiv \mathcal{I}_{0}$, reflecting the fact that we start from a deterministic word $u$. Suppose $I=\left\langle a_{0} p_{0} \cdots a_{n} p_{n}\right\rangle \in \mathcal{I}_{n}^{\omega}$ for some $n \in \mathbb{N}_{0}$ and $\omega_{I}=v_{1} \cdots v_{k}$, with $k \in \mathbb{N}$. The set of all individuals produced by $I$ is given by $A_{I}=\left\{\left\langle a_{0} p_{0} \cdots a_{n} p_{n} v_{j} j\right\rangle\right.$ : $j \in\{1, \ldots, k\}\}$ and we define $\mathcal{I}_{n+1}^{\omega}=\cup_{I \in \mathcal{I}_{n}^{\omega}} A_{I}$.

In the next step, we construct a word from each of the sets $\mathcal{I}_{n}^{\omega}$ that we call a level-n inflation word of the initial word $u$. For $n \in \mathbb{N}_{0}$, we define a strict total order relation $<$ on $\mathcal{I}_{n}^{\omega}$ as follows. We set $\left\langle a_{0} p_{0} \cdots a_{n} p_{n}\right\rangle<\left\langle a_{0}^{\prime} p_{0}^{\prime} \cdots a_{n}^{\prime} p_{n}^{\prime}\right\rangle$ if there is an $i \in\{0, \ldots, n\}$ such that $p_{i}<p_{i}^{\prime}$ 


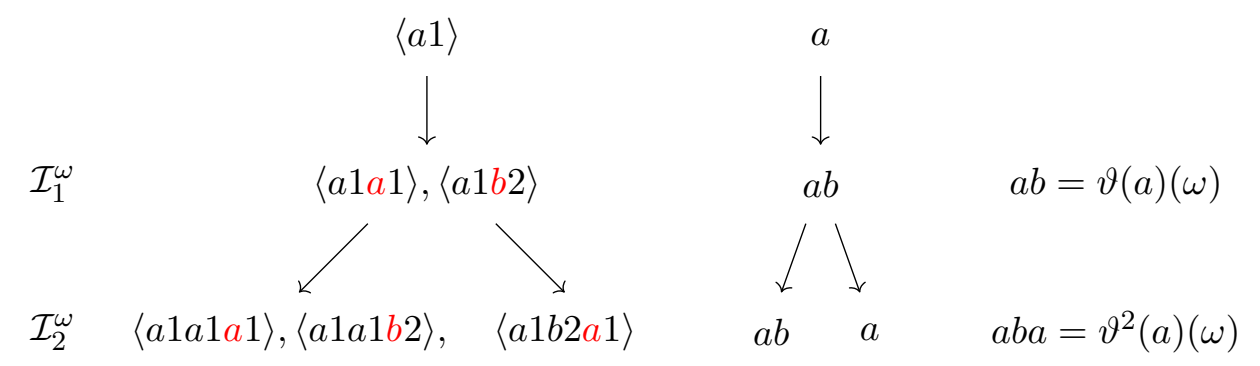

FiguRE 6. Left hand side: Illustration of the selected individuals of the first two generations constructed from $\omega$ in Example 7.3. The types of the individuals are colored in red. The tree on the right displays the choices of $\omega_{I}$ for $I=\langle a 1\rangle$ (second line) and for $I \in \mathcal{I}_{1}^{\omega}$ (third line).

and $p_{j}=p_{j}^{\prime}$ for all $j<i$. Suppose $I_{n}^{1}<I_{n}^{2}<\ldots<I_{n}^{r}$ is the ordering of the individuals in $\mathcal{I}_{n}^{\omega}$. Then, for every $n \in \mathbb{N}_{0}$,

$$
\vartheta^{n+1}(u): \Omega_{u} \rightarrow \mathcal{A}^{+}, \quad \vartheta^{n+1}(u)(\omega):=\omega_{I_{n}^{1}} \cdots \omega_{I_{n}^{r}} .
$$

Note that we have suppressed the dependence of $I_{n}^{j}$ on $\omega$ for notational convenience. It is worth noticing that if $\vartheta^{n+1}(u)(\omega)=v_{1} \cdots v_{s}$, then $s=\operatorname{card} \mathcal{I}_{n+1}^{\omega}$ and $v_{j}$ is the type of $I_{n+1}^{j}$ for $j \in\{1, \ldots, s\}$.

Lemma 7.2. The sequence $\left(\vartheta^{n}(u)\right)_{n \in \mathbb{N}}$ forms a stationary Markov chain with transition kernel

$$
\mathbb{P}_{u}\left[\vartheta^{n+1}(u)=w \mid \vartheta^{n}(u)=v\right]=P(v, w),
$$

for all $n \in \mathbb{N}$ and $v, w \in \mathcal{A}^{+}$.

The proof is analogous to the one presented for Lemma 7.1 and is left to the interested reader.

Example 7.3. Consider the random Fibonacci substitution rule on $\mathcal{A}=\{a, b\}$, given by $P_{b}^{\vartheta}=\delta_{a}$ and $P_{a}^{\vartheta}=p \delta_{a b}+q \delta_{b a}$, with $p \in(0,1)$ and $q=1-p$. Let us start from the initial word $u=a$, so $\mathcal{I}_{0}=\{\langle a 1\rangle\}$. Suppose $\omega=\left(\omega_{I}\right)_{I \in \mathcal{I}} \in \Omega_{a}$, with $\omega_{\langle a 1\rangle}=a b$. Then, $\mathcal{I}_{1}^{\omega}=$ $\{\langle a 1 a 1\rangle,\langle a 1 b 2\rangle\}$. These are ordered as $\langle a 1 a 1\rangle<\langle a 1 b 2\rangle$. Suppose further $\omega_{\langle a 1 a 1\rangle}=a b$ and $\omega_{\langle a 1 b 2\rangle}=a$. Consequently, $\vartheta^{2}(a)(\omega)=a b a$; compare Figure 7 for a graphical interpretation. On the other hand $\omega^{\prime}=\left(\omega_{I}^{\prime}\right)_{I \in \mathcal{I}} \in \Omega$ with $\omega_{\langle a 1\rangle}^{\prime}=b a, \omega_{\langle a 1 b 1\rangle}^{\prime}=a, \omega_{\langle a 1 a 2\rangle}^{\prime}=b a$ yields again $\vartheta^{2}(a)\left(\omega^{\prime}\right)=a b a$. Since these are the only ways to obtain the word $a b a$ in two steps with non-vanishing probability, we obtain

$$
\begin{aligned}
\mathbb{P}_{a}\left[\vartheta^{2}(a)=a b a\right]= & \mathbb{P}_{a}\left[\omega_{\langle a 1\rangle}=a b \wedge \omega_{\langle a 1 a 1\rangle}=a b \wedge \omega_{\langle a 1 b 2\rangle}=a\right] \\
& +\mathbb{P}_{a}\left[\omega_{\langle a 1\rangle}=b a \wedge \omega_{\langle a 1 b 1\rangle}=a \wedge \omega_{\langle a 1 a 2\rangle}=b a\right]=p^{2}+q^{2} .
\end{aligned}
$$

Given $u=u_{1} \cdots u_{m}$, we will next discuss how to embed random variables $\vartheta^{n}\left(u_{j}\right)$ for $j \in$ $\{1, \ldots, m\}$ into the probability space $\left(\Omega_{u}, \mathcal{F}_{u}, \mathbb{P}_{u}\right)$. Note that we can partition $\mathcal{I}=\cup_{j=1}^{m} \mathcal{I}(j)$ with $I \in \mathcal{I}(j)$ if and only if $I$ is of the form $I=\left\langle u_{j} j \cdots\right\rangle$. This leads to a decomposition $\Omega_{u}=\Omega_{1} \times \cdots \times \Omega_{m}$, as well as $\mathcal{B}_{u}=\mathcal{B}_{1} \otimes \cdots \otimes \mathcal{B}_{m}$ and $\mathbb{P}_{u}=\mathbb{P}_{1} \otimes \cdots \otimes P_{m}$, where 
$\left(\Omega_{j}, \mathcal{B}_{j}, \mathbb{P}_{j}\right)$ is the probability space constructed from $\mathcal{I}(j)$ for all $j \in\{1, \ldots, m\}$. Note that $\left(\Omega_{j}, \mathcal{B}_{j}, \mathbb{P}_{j}\right)$ is just a copy of the probability space $\left(\Omega_{u_{j}}, \mathcal{B}_{u_{j}}, \mathbb{P}_{u_{j}}\right)$. Thus, $\vartheta^{n}\left(u_{j}\right)$ is well-defined on $\left(\Omega_{j}, \mathcal{B}_{j}, \mathbb{P}_{j}\right)$ and fulfils the defining relations of the corresponding Markov chain. For $\omega=\left(\omega_{1}, \ldots, \omega_{m}\right) \in \Omega_{u}$, we define $\vartheta^{n}\left(u_{j}\right)(\omega)=\vartheta^{n}\left(u_{j}\right)\left(\omega_{j}\right)$ for all $j \in\{1, \ldots, m\}$. Because of the product structure of $\left(\Omega_{u}, \mathcal{B}_{u}, \mathbb{P}_{u}\right)$, the random variables $\vartheta^{n}\left(u_{j}\right)$ and $\vartheta^{n}\left(u_{k}\right)$ are independent for every $n \in \mathbb{N}$ and $j \neq k$. Finally note that for $\omega \in \Omega_{u}, \mathcal{I}_{n}^{\omega}=\cup_{j=1}^{m}\left(\mathcal{I}_{n}^{\omega} \cap \mathcal{I}(j)\right)$ and for $j<k$ all the elements in $\mathcal{I}_{n}^{\omega} \cap \mathcal{I}(j)$ are smaller than those in $\mathcal{I}_{n}^{\omega} \cap \mathcal{I}(k)$. Since $\vartheta^{n}\left(u_{j}\right)$ depends only on the individuals in $\mathcal{I}_{n-1}^{\omega} \cap \mathcal{I}(j)$, this implies

$$
\vartheta^{n}(u)(\omega)=\left(\vartheta^{n}\left(u_{1}\right) \cdots \vartheta^{n}\left(u_{m}\right)\right)(\omega)
$$

for all $\omega \in \Omega_{u}$ and $n \in \mathbb{N}$.

\section{ACKNOWLEDGEMENTS}

It is a pleasure to thank Michael Baake and Dan Rust for helpful discussions.

This work is supported by the German Research Foundation (DFG) via the Collaborative Research Centre (CRC 1283) through the faculty of Mathematics, Bielefeld University. Also,

PG was partially supported by the Research Centre of Mathematical Modelling $\left(\mathrm{RCM}^{2}\right)$ of Bielefeld University, while TS was also supported by NSERC via grant 03762-2014.

\section{REFERENCES}

[1] K.B. Athreya and P. Ney, Branching Processes, Springer, Berlin (1972).

[2] M. Baake and U. Grimm, Aperiodic Order. Vol. 1: A Mathematical Invitation, Cambridge University Press, Cambridge (2013).

[3] M. Baake and D. Lenz, Dynamical systems on translation bounded measures: Pure point dynamical and diffraction spectra, Ergodic Th. \& Dynam. Syst. 24 (2004), 1867-1893; arXiv:math.DS/0302231.

[4] M. Baake, T. Spindeler and N. Strungaru, Diffraction of compatible random substitutions in one dimension, Indag. Math. 29 (2018), 1031-1071; arXiv:1712.00323.

[5] D. Damanik, M. Embree and A. Gorodetski, Spectral properties of Schrödinger operators arising in the study of quasicrystals, in Mathematics of Aperiodic Order, J. Kellendonk, D. Lenz and J. Savinien (eds.), Birkhäuser, Basel (2015), 307-370; arXiv:1210.5753.

[6] M. Denker, C. Grillenberger and K. Sigmund, Ergodic Theory on Compact Spaces, Springer, Berlin (1976).

[7] C. Godrèche and J.M. Luck, Quasiperiodicity and randomness in tilings of the plane, J. Stat. Phys. 55 (1989), 1-28.

[8] P. Gohlke, Inflation word entropy for semi-compatible random substitutions, in preparation.

[9] P. Gohlke, On a family of semi-compatible random substitutions Masters thesis, Univ. Bielefeld (2017);

[10] P. Gohlke, D. Rust and T. Spindeler, Shifts of finite type and random substitutions, Discrete Contin. Dyn. Syst. (forthcoming); arXiv:1712.05340.

[11] F. den Hollander, Large Deviations, AMS, Providence, RI (2000).

[12] Y. Hu, D. Tian and L. Wang, Renormalization group approach to the random period doubling lattice, Phys. Lett. A 207 (1995), 293-298.

[13] D. Koslicki, Substitution Markov chains with applications to molecular evolution, PhD thesis, The Pennsylvania State University (2012).

[14] W. Krieger, On the uniqueness of the equilibrium state, Math. Systems Theory 8 (1974), 97-104.

[15] W. Li, Spatial l/f Spectra in Open Dynamical Systems, Europhys. Lett. 10 (1989), 395-400.

[16] C. Maldonado, L. Trejo-Valencia and E. Ugalde, Constant-length random substitutions and Gibbs measures, J. Stat. Phys. 171 (2018), 269-287; arXiv:1712.00586. 
[17] R. Mansilla and G. Cocho, Multiscaling in expansion-modification systems: An explanation for long range correlation in DNA, Complex Systems 12 (2000), 207-240.

[18] C.J. Mode, Multitype Branching Processes: Theory and Applications, Elsevier, New York (1971).

[19] M. Moll, Diffraction of random noble means words, J. Stat. Phys. 156 (2014), 1221-1236.

[20] M. Moll, On a Family of Random Noble Means Substitutions, PhD thesis, Univ. Bielefeld (2013); available at https://pub.uni-bielefeld.de/publication/2637807.

[21] W. Parry, Intrinsic Markov chains, Trans. Amer. Math. Soc. 112 (1964), 55-66.

[22] K.R. Parthasarathy, Introduction to Probability and Measure, Macmillan, New Delhi (2005).

[23] J. Peyrière, Substitutions aléatoires itérées, Sémin. Théor. Nombres Bordeaux 17 (1980-1981), 1-9.

[24] N. Pytheas Fogg, Substitutions in Dynamics, Arithmetics and Combinatorics, LNM 1794, Springer, Berlin (2002).

[25] M. Queffélec, Substitution Dynamical Systems-Spectral Analysis, 2nd ed., LNM 1294, Springer, Berlin (2010).

[26] G. Rozenberg and A. Salomaa, The Mathematical Theory of L Systems, Academic Press, New York (1980).

[27] D. Rust, Periodic points in random substitution subshifts, preprint arXiv:1808.05934.

[28] D. Rust and T. Spindeler, Dynamical systems arising from random substitutions, Indag. Math. 29 (2018), 1131-1155; arXiv: 1707.09836.

[29] R. Salgado-García and E. Ugalde, Exact scaling in the expansion-modification system, J. Stat. Phys. 153 (2013), 842-863; arXiv: 1202.2549.

[30] E. Seneta, Non-negative Matrices, Allen \& Unwin, London (1973).

[31] P. Walters, An Introduction to Ergodic Theory, GTM 79, Springer, New York (2000).

[32] M.A. Zaks, Multifractal Fourier spectra and power-law decay of correlations in random substitution sequences, Phys. Rev. E 65 (2001), 011111 (5 pp.).

FAKUltät FÜr MAThematik, Universität Bielefeld, Postfach 100131, 33501 Bielefeld, Germany

E-mail address: pgohlke@math.uni-bielefeld.de

Department of Mathematical and Statistical Sciences, 632 CAB, University of Alberta, Edmonton, AB, T6G 2G1, Canada

E-mail address: spindele@ualberta.ca 